\title{
Intersecting subvarieties of abelian varieties with algebraic subgroups of complementary dimension
}

\section{Journal Article}

\section{Author(s):}

Habegger, P.

Publication date:

2009-05

\section{Permanent link:}

https://doi.org/10.3929/ethz-b-000156804

\section{Rights / license:}

In Copyright - Non-Commercial Use Permitted

\section{Originally published in:}

Inventiones mathematicae 176(2), https://doi.org/10.1007/s00222-008-0170-6 


\section{Intersecting subvarieties of abelian varieties with algebraic subgroups of complementary dimension}

\section{P. Habegger}

ETH Zürich, Departement Mathematik, Rämistrasse 101, 8092 Zürich, Switzerland (e-mail: habegger@math.ethz.ch)

Oblatum 9-VII-2008 \& 12-XI-2008

Published online: 16 December 2008 - (c) Springer-Verlag 2008

\section{Introduction}

Conjectures on the intersection of an irreducible closed subvariety $X$ of a semi-abelian variety $S$, both defined over $\mathbf{C}$, with the union of all algebraic subgroups with restricted codimension were stated independently and in somewhat different form by Zilber [27] and Pink [17]. The following statement would be a common consequence of these conjectures:

Conjecture 1. Let $X$ be an irreducible closed subvariety of a semi-abelian variety $S$, both defined over $\mathbf{C}$. If $X$ is not contained in a proper algebraic subgroup of $S$, then the set of points in $X(\mathbf{C})$ contained in the union of all algebraic subgroups of $S$ with codimension at least $1+\operatorname{dim} X$ is not Zariski dense in $X$.

In fact both authors stated stronger conjectures. Zilber conjectured a finiteness statement for subvarieties that are contained in an improper intersection of $X$ with an algebraic subgroup of $S$. Pink was motivated by a more general conjecture in the context of mixed Shimura varieties. He also showed that Conjecture 1 implies the celebrated Mordell-Lang conjecture.

An important precursor was set in 1999 by Bombieri, Masser, and Zannier [3] who proved the following result for an algebraic curve $C$ embedded in the algebraic torus $\mathbf{G}_{m}^{n}$, not contained in the translate of a proper algebraic subgroup, and defined over $\overline{\mathbf{Q}}$, an algebraic closure of $\mathbf{Q}$ : the set of algebraic points of $C$ which are contained in the union of all algebraic subgroups of codimension at least 1 has bounded absolute Weil height. They then used their result to show that there are only finitely many points on $C$ contained in the union of all algebraic subgroups of codimension at least 2. 
Later, they conjectured (bounded height conjecture [4]) the existence of a height bound in connection with the intersection of an irreducible subvariety of $\mathbf{G}_{m}^{n}$ with the union of all algebraic subgroups whose codimension is at least the dimension of the variety. This conjecture and its abelian analogue were only known for subvarieties of small dimension or codimension; further down we will state it more precisely and give a short survey on what is known.

The main result of this paper, the bounded height theorem stated below, is a proof of this conjecture in the case where $\mathbf{G}_{m}^{n}$ is replaced by any abelian variety defined over $\overline{\mathbf{Q}}$. We also show two corollaries which imply finiteness, the second of which shows that Conjecture 1 holds for generic subvarieties of an abelian variety with complex multiplication when all data is defined over $\overline{\mathbf{Q}}$.

For a semi-abelian variety $S$ defined over $\mathbf{C}$ and an integer $s$ with $s \leq$ $\operatorname{dim} S$ we define

$$
S^{[s]}=\bigcup_{\operatorname{codim} H \geq s} H(\mathbf{C}),
$$

where the union is taken over all algebraic subgroups of $S$ with codimension at least $s$. If $s>\operatorname{dim} S$ we set $S^{[s]}=\emptyset$, the empty set. For example by Bombieri, Masser, and Zannier's result $C(\overline{\mathbf{Q}}) \cap\left(\mathbf{G}_{m}^{n}\right)^{[1]}$ has bounded height and $C(\overline{\mathbf{Q}}) \cap\left(\mathbf{G}_{m}^{n}\right)^{[2]}$ is finite.

Before we state the main result we shall define a natural subset of $X$ for which we will be able to prove the height bound. For an integer $s$ we define $X^{\mathrm{oa},[s]}$ to be $X(\mathbf{C})$ deprived of all $Y(\mathbf{C})$ where $Y \subset X$ is an irreducible closed subvariety such that there exists a coset $H$, i.e. the translate of an algebraic subgroup of $S$, with $Y \subset H$ and

$$
\operatorname{dim} Y \geq \max \{1, s+\operatorname{dim} H-\operatorname{dim} S+1\} .
$$

We abbreviate $X^{\mathrm{oa}}=X^{\mathrm{oa},[\operatorname{dim} X]}$ and also set $X^{\mathrm{oa},[s]}(\overline{\mathbf{Q}})=X^{\mathrm{oa},[s]} \cap X(\overline{\mathbf{Q}})$ and $X^{\mathrm{oa}}(\overline{\mathbf{Q}})=X^{\mathrm{oa},[\operatorname{dim} X]}(\overline{\mathbf{Q}})$. These so-called anomalous subvarieties were essentially introduced by Bombieri, Masser, and Zannier [4,6]. For $S=\mathbf{G}_{m}^{n}$ they first proved [4] that $X^{\mathrm{oa}}$ is Zariski open (but possibly empty) in $X$ and later [6] the same for the more general $X^{\mathrm{oa},[s]}$. For $S$ an abelian variety, Rémond [21] showed that $X^{\mathrm{oa},[s]}$ is Zariski open (in his notation $X^{\mathrm{oa},[s]}=$ $X \backslash Z_{X \text {,an }}^{(1+s)}$ ). This result is not used in the proof of our main result below.

In special cases, $X^{\mathrm{oa},[s]}$ is easy to determine. If $s \leq \operatorname{dim} X-1$ then $X^{\mathrm{oa},[s]}=\varnothing$ (at least if $\left.\operatorname{dim} X \geq 1\right)$; indeed one may take $H=S$ and $Y=X$. For sufficiently general $X$ one expects that $X^{\text {oa }}$ is non-empty and hence Zariski dense. We will not make this statement precise, but a criterion is given in Corollary 2. In the other extreme we always have $X^{\text {oa, }[\operatorname{dim} S]}=X$. Finally, if $X$ is a curve, then $X^{\mathrm{oa},[s]}=\emptyset$ if and only if $X$ is contained in a coset of codimension at least $s$ and $X^{\mathrm{oa},[s]}=X$ otherwise.

For general semi-abelian varieties one could conjecture that if $S$ and $X$ are both defined over $\overline{\mathbf{Q}}$ and after choosing a suitable height on $S(\overline{\mathbf{Q}})$ then 
the set $X^{\text {oa, }[s]}(\overline{\mathbf{Q}}) \cap S^{[s]}$ has bounded height. By the comment above, this statement is trivial if $s \leq \operatorname{dim} X-1$. In the critical case $s=\operatorname{dim} X$, i.e. when the subvariety and algebraic subgroups have complementary dimension, Bombieri, Masser, and Zannier (bounded height conjecture [4]) stated this conjecture for $S=\mathbf{G}_{m}^{n}$.

From now on let $A$ be an abelian variety and let $X \subset A$ be an irreducible closed subvariety, both defined over $\overline{\mathbf{Q}}$. Our method allows us to treat not only points on $X$ contained in the union of all algebraic subgroups of restricted codimension, but also those which are in a sense close to this union. To make this precise, let $\hat{h}: A(\overline{\mathbf{Q}}) \rightarrow[0, \infty)$ be the Néron-Tate height associated to a symmetric ample line bundle on $A$ (cf. Sect. 2). In particular, the height of the unit element of $A$ is zero. Let $\Sigma \subset A(\mathbf{C})$ be a subset and let $\epsilon \geq 0$, we define the "truncated cone" around $\Sigma$ as

$$
\begin{aligned}
\mathcal{C}(\Sigma, \epsilon)=\{a+b ; a \in \Sigma \cap A(\overline{\mathbf{Q}}) \text { and } b \in & A(\overline{\mathbf{Q}}) \\
& \text { with } \hat{h}(b) \leq \epsilon(1+\hat{h}(a))\} .
\end{aligned}
$$

We are now ready to formulate the bounded height theorem.

Theorem. Let $A$ be an abelian variety and let $X$ be an irreducible closed subvariety of A, both defined over $\overline{\mathbf{Q}}$. Let us consider the Néron-Tate height on $A(\overline{\mathbf{Q}})$ associated to a fixed symmetric and ample line bundle. If $s$ is an integer there exists $\epsilon>0$ such that the height is bounded above on $X^{\mathrm{oa},[s]}(\overline{\mathbf{Q}}) \cap \mathcal{C}\left(A^{[s]}, \epsilon\right)$.

Special cases of this result have already appeared in literature. For example Viada [23] proved boundedness of height when $s=1$ (and $\epsilon=0$ ) if $X$ is a curve and $A$ is a power of an elliptic curve. The case of a curve in any abelian variety with $s=1$ and $\epsilon=0$ was proved by Rémond [19]. He also showed [21] (cf. [20] for a result involving an $\epsilon$ ) that if $\Gamma \subset A(\overline{\mathbf{Q}})$ is a subgroup of finite rank, then $X^{\mathrm{oa},[s]}(\overline{\mathbf{Q}}) \cap\left(\Gamma+A^{[1+s]}\right)$ has bounded height. The proof involves his generalized Vojta inequality and is independent from ours. Both his approach and ours have at least one common factor: the use of Ax's theorem [1]. In the multiplicative setting boundedness of height was demonstrated for curves as already discussed above, for planes [5] by Bombieri, Masser, and Zannier, and for hypersurfaces [26] by Bombieri and Zannier. Using an approach related to the one employed in this paper, the author has obtained a proof of the bounded height conjecture for all subvarieties of $\mathbf{G}_{m}^{n}$ [13].

Already Bombieri, Masser, and Zannier [3] observed that a height bound result implies a finiteness result if a sufficiently strong relative Lehmertype height lower bound is available. In the context of algebraic tori, such a bound was proved by Amoroso and David. Ratazzi [18] showed a semirelative Lehmer-type height lower bound for abelian varieties with complex multiplication. His result was recently improved to a relative Lehmer-type 
height lower bound by Carrizosa [8] which suffices to deduce the following corollary.

Corollary 1. Let A be an abelian variety with complex multiplication defined over $\overline{\mathbf{Q}}$ and let $X \subset A$ be an irreducible closed subvariety also defined over $\overline{\mathbf{Q}}$. Then $X^{\mathrm{oa},[s]}(\overline{\mathbf{Q}}) \cap A^{[1+s]}$ is finite.

The next corollary is a reformulation of Corollary 1 using Rémond's result that $X^{\mathrm{oa},[s]}$ is Zariski open; cf. his finiteness result involving a finitely generated subgroup [21].

Corollary 2. Let $A$ be an abelian variety with complex multiplication defined over $\overline{\mathbf{Q}}$ and let $X \subset A$ be an irreducible closed subvariety of positive dimension also defined over $\overline{\mathbf{Q}}$. We assume that for all abelian varieties $B$ and all surjective homomorphisms of algebraic groups $\varphi: A \rightarrow B$ we have $\operatorname{dim} \varphi(X)=\min \{\operatorname{dim} X, \operatorname{dim} B\}$. Then $X^{\text {oa }}$ is non-empty and $X(\overline{\mathbf{Q}}) \cap A^{[1+\operatorname{dim} X]}$ is not Zariski dense in $X$.

So under an additional geometric hypothesis on $X$, the corollary above implies Conjecture 1 if the ambient abelian variety has complex multiplication and if everything is defined over $\overline{\mathbf{Q}}$.

More recently, a new approach to obtain finiteness using an effective Bogomolov-type height lower bound was worked out independently by Viada in the abelian case and by the author in the multiplicative one. In certain cases such an approach has the advantage of being able to replace $A^{[1+\operatorname{dim} X]}$ in Corollary 1 by $\mathcal{C}\left(A^{[1+\operatorname{dim} X]}, \epsilon\right)$ for some positive $\epsilon$; thereby providing a connection between Conjecture 1 and the Bogomolov conjecture.

We now give an overview of the proof of the bounded height theorem (with $\epsilon=0$ ). Let $A$ and $X$ be as in the theorem, certainly we may assume $X^{\mathrm{oa},[s]} \neq \emptyset$. One can show that there is a finite set of abelian varieties of dimension at least $s$ such that any $p \in A^{[s]}$ lies in the kernel of a surjective homomorphism $A \rightarrow B$ with $B$ from this set. Hence let $\psi: A \rightarrow B$ be any such morphism. The proof of the theorem is based on a lower bound, cf. Lemma 7, for the height of $\psi(p)$ in terms of the height of $p$ for generic $p \in X(\overline{\mathbf{Q}})$. This lemma applies a theorem of Siu to prove the existence of a non-zero global section of a certain line bundle. The bound is linear in the height of $p$ with non-negative leading term $c(\psi)$ depending on a certain intersection number determined by $\psi$. Let us assume for the moment that $c(\psi)$ can be bounded from below by a positive constant which is independent of $\psi$. If $\psi(p)=0$, the unit element, then the height of $p$ is bounded from above in function of the constant term from Lemma 7. This term might depend on $\psi$, but by a simple result from diophantine approximation we can approximate $\psi$ by a new, essentially simpler, surjective homomorphism $A \rightarrow B$. We then obtain a bound for the height of $p$ which is independent of $\psi$. This then quickly leads to the desired height upper bound. 
How does one bound $c(\psi)$ from below? Using the theorem of the cube we extend the definition of $c(\psi)$ to the finite dimensional vector space $\operatorname{Hom}(A, B) \otimes_{\mathbf{Z}} \mathbf{R}$ and obtain a continuous map with values in [0, $\left.\infty\right)$. A large portion of this paper consists in proving $c(\psi)>0$ if $\psi \in \operatorname{Hom}(A, B) \otimes_{\mathbf{z}} \mathbf{R}$ has maximal rank; this is where $X^{\mathrm{oa},[s]} \neq \emptyset$ is used. Such a positivity statement is derived in Proposition 5 which we discuss separately further down. By continuity $c(\psi)$ is then bounded from below uniformly by a positive constant for all $\psi$ in a fixed compact subspace $\mathcal{K} \subset \operatorname{Hom}(A, B) \otimes_{\mathbf{Z}} \mathbf{R}$ consisting only of elements of maximal rank. It is then not difficult to extend this result to all $\psi$ with $\psi / \lambda \in \mathcal{K}$ for some $\lambda \geq 1$. If no such $\psi / \lambda$ is in $\mathcal{K}$ we can exploit the left-action of $\operatorname{End}(B)^{*}$ on $\operatorname{Hom}(A, B)$ to prove suitable lower bound for $c(\psi)$, at least if $\mathcal{K}$ was chosen suitably.

As promised we discuss the positivity result $c(\psi)>0$ which is central to the proof of the bounded height theorem. An element $\psi \in \operatorname{Hom}(A, B) \otimes_{\mathbf{Z}} \mathbf{R}$ may not define a morphism of varieties, but it does define a linear map $T(A) \rightarrow T(B)$ between holomorphic tangent spaces of $A$ and $B$ at the unit element. The preimage of the non-singular locus of $X(\mathbf{C})$ under the exponential map $T(A) \rightarrow A(\mathbf{C})$ is a complex submanifold of $T(A)$. And it is in this context where we can make sense of $\psi$. Let us assume $\psi$ has maximal rank and $c(\psi)=0$. Then we combine an estimate for the number of periods of $B$ contained in a certain (possibly non-convex) subset of $T(B)$ together with a theorem of $\mathrm{Ax}$ to show $X^{\mathrm{oa},[\mathrm{dim} B]}=\emptyset$. But this contradicts our initial hypothesis since $X^{\mathrm{oa},[s]} \subset X^{\mathrm{oa},[\mathrm{dim} B]}$.

The proof of the theorem applies basic results from intersection theory such as the projection formula; so we use the fact that abelian varieties are complete in an essential way. The proof does not work unchanged for non-complete semi-abelian varieties. Although many tools, such as Ax's theorem, are applicable in this more general setting, some, such as the theorem of the cube, are not. For this reason we investigate the multiplicative case in a separate article already mentioned above. Future work will be devoted to more general semi-abelian cases.

The article is organized as follows. In Sect. 2 we recall some basic facts on the height and Néron-Tate height associated to a line bundle. Sections 3 and 4 contain the argument involving diophantine approximation. In Sect. 5 we use Siu's theorem and show Proposition 1, a weak version of the bounded height theorem under an additional hypothesis on $X$ which amounts to the inequality $c(\psi)>0$ discussed above. In order to clarify the remainder of the demonstration we present a proof of a weaker variant in Sect. 6 by assuming that $A$ is a power of an elliptic curve without complex multiplication. At the end of this section we try to convince the reader why a proof of our main result for varieties of arbitrary dimension must differ substantially from a proof for curves or hypersurfaces. Section 7 is reserved for the lattice point counting result mentioned above. This is applied in Sects. 8 and 9 together with Ax's theorem of prove a positivity condition for $c(\psi)$. In Sect. 10 we complete the proof of the bounded height theorem and prove Corollaries 1 and 2. 
I am indepted to David Masser for helpful comments on this article and for raising Ax's paper [1] to my attention. I also thank Gaël Rémond for sending me a preliminary version of his paper [21] and the referee for making many suggestions which led to improvements of the manuscript.

\section{On heights}

In this section we recall some properties of heights on algebraic varieties. Therefore, all varieties in this section shall be defined over $\overline{\mathbf{Q}}$ unless stated otherwise.

We call two maps from a common set to $\mathbf{R}$ equivalent if the absolute value of their difference is bounded. The set of equivalence classes has the structure of an $\mathbf{R}$-vector space. If $h$ and $h^{\prime}$ are two such classes we say that $h^{\prime}$ is bounded below by $h$, or $h \leq h^{\prime}$, if the difference of some representative of $h^{\prime}$ and some representative of $h$ is bounded from below.

Let $X$ be an irreducible projective variety defined over $\mathbf{C}$ and let $\operatorname{Pic}(X)$ denote the Picard group of $X$. Elements of $\operatorname{Pic}(X)$ correspond to isomorphism classes of line bundles on $X$ and we will usually identify a line bundle with its isomorphism class.

Now assume $X$ is defined over $\overline{\mathbf{Q}}$. Any $\mathcal{L} \in \operatorname{Pic}(X)$ determines an equivalence class of maps $X(\overline{\mathbf{Q}}) \rightarrow \mathbf{R}$ called the height on $X$ associated to $\mathcal{L}$. We denote this equivalence class with $h_{X, \mathcal{L}}$. A construction is given by [2, Theorem 2.3.8, p. 41].

The height has the following functional properties: if $Y$ is an another irreducible projective variety, also defined over $\overline{\mathbf{Q}}$, and $f: Y \rightarrow X$ is a morphism, then

$$
h_{Y, f^{*} \mathcal{L}}=h_{X, \mathcal{L}} \circ f
$$

(equality as equivalence classes). Furthermore, if $\mathcal{M} \in \operatorname{Pic}(X)$ then

$$
h_{X, \mathcal{L} \otimes \mathcal{M}}=h_{X, \mathcal{L}}+h_{X, \mathcal{M}} .
$$

Finally, the height is gauged as follows: if $\mathcal{O}(1)$ is the unique ample generator of the Picard group of $n$-dimensional projective space $\mathbf{P}^{n}$, then the absolute logarithmic Weil height lies in $h_{\mathbf{P}^{n}, \mathcal{O}(1)}$. The absolute logarithmic Weil height is never negative and in particular bounded from below. This need not be true for a representative of $h_{X, \mathcal{L}}$. But if $\mathcal{L}$ has a non-zero global section, then there exists a Zariski open non-empty $U \subset X$ such that

$$
\text { any representative of } h_{X, \mathcal{L}} \text { is bounded from below on } U(\overline{\mathbf{Q}}) \text {. }
$$

It follows from (2) that (3) already holds if some positive power of $\mathcal{L}$ has a non-zero global section. If for any point of $X$ we can find a global section of $\mathcal{L}$ not vanishing there, then we can take $U=X$ in (3), this is showed in $[2$, Proposition 2.3 .9$, p. 42]. For example, very ample line bundles have this property. It follows from (2) that (3) holds with $U=X$ if we merely assume that $\mathcal{L}$ is ample. 
We deduce a useful height inequality involving morphisms. Let $\mathcal{M}, \mathcal{N} \in$ $\operatorname{Pic}(Y)$ and let us suppose that $\mathcal{M}$ is ample. Then there exists a positive integer $C$ such that $\mathcal{M}^{\otimes C} \otimes \mathcal{N}^{\otimes(-1)}$ is also ample. Switching to heights and using the functional properties we get

$$
h_{Y, \mathcal{N}} \leq C h_{Y, \mathcal{M}} .
$$

Let $f: Y \rightarrow X$ be a morphism. We apply (4) to $\mathcal{N}=f^{*} \mathcal{L}$ where $\mathcal{L} \in$ $\operatorname{Pic}(X)$ is arbitrary. Using (1) we deduce

$$
h_{X, \mathfrak{L}} \circ f \leq C h_{Y, \mathcal{M}} .
$$

Next we consider heights on abelian varieties. First, let us assume that $A$ is an abelian variety defined over $\mathbf{C}$. Any $n \in \mathbf{Z}$ defines the multiplication by $n$ morphism $[n]: A \rightarrow A$. We call $\mathcal{L} \in \operatorname{Pic}(A)$ symmetric if $[-1]^{*} \mathscr{L}=\mathscr{L}$, in this case it is well-known that $[n]^{*} \mathcal{L}=\mathscr{L}^{\otimes n^{2}}$. Now say $A$ is defined over $\overline{\mathbf{Q}}$. Then as above we can associate an equivalence class of maps $A(\overline{\mathbf{Q}}) \rightarrow \mathbf{R}$ to any $\mathcal{L} \in \operatorname{Pic}(A)$. But now we get more information because of the presence of a group structure. We assume that $\mathcal{L}$ is symmetric. Using Tate's limit argument we assign to $\mathcal{L}$ a well-defined map $\hat{h}_{A, \mathcal{L}}: A(\overline{\mathbf{Q}}) \rightarrow \mathbf{R}$ called the Néron-Tate height associated to $\mathcal{L}$, or the Néron-Tate height, or simply the height. Then $\hat{h}_{A, \mathcal{L}}$ is a quadratic form; in particular $\hat{h}_{A, \mathcal{L}}(n p)=n^{2} \hat{h}_{A, \mathcal{L}}(p)$ for all $n \in \mathbf{Z}$ and $p \in A(\overline{\mathbf{Q}})$, making the Néron-Tate height homogeneous of degree 2. We collect some useful functional properties. A basic fact is that

$$
\hat{h}_{A, \mathscr{L}} \text { lies in the equivalence class } h_{A, \mathcal{L}} \text {. }
$$

If $B$ is another abelian variety defined over $\overline{\mathbf{Q}}$ and $f: B \rightarrow A$ is a homomorphism of algebraic groups (in other words, $f$ is a morphism of varieties with $f(0)=0)$ then $f^{*} \mathcal{L}$ is symmetric and

$$
\hat{h}_{B, f^{*} \mathcal{L}}=\hat{h}_{A, \mathcal{L}} \circ f .
$$

Furthermore, if $\mathcal{M} \in \operatorname{Pic}(A)$ is also symmetric, then so is $\mathcal{L} \otimes \mathcal{M}$ and we have

$$
\hat{h}_{A, \mathcal{L} \otimes \mathcal{M}}=\hat{h}_{A, \mathscr{L}}+\hat{h}_{A, \mathcal{M}} .
$$

So, for abelian varieties the height is no longer just an equivalence class of maps but a real map, moreover the functional properties hold strictly. These will be used freely throughout the paper.

Let $\mathcal{L}$ be symmetric and ample. Then $\hat{h}_{A, \mathcal{L}}(p) \geq 0$ for $p \in A(\overline{\mathbf{Q}})$ with equality if and only if $p$ is torsion. This is usually referred to as Kronecker's theorem. Another useful inequality which follows from the fact that $\hat{h}_{A, \mathscr{L}}$ is a non-negative quadratic form is

$$
\hat{h}_{A, \mathcal{L}}\left(p+p^{\prime}\right) \leq 2 \hat{h}_{A, \mathcal{L}}(p)+2 \hat{h}_{A, \mathcal{L}}\left(p^{\prime}\right)
$$

for all $p, p^{\prime} \in A(\overline{\mathbf{Q}})$. 
It is well-known that $\operatorname{Hom}(B, A)$, the $\mathbf{Z}$-module of homomorphisms $B \rightarrow A$, is finitely generated and free. Let $\|\cdot\|$ be any norm on the finite dimensional $\mathbf{R}$-vector space $\operatorname{Hom}(B, A) \otimes_{\mathbf{Z}} \mathbf{R}$. Let $\mathcal{M} \in \operatorname{Pic}(B)$ be symmetric and ample and let $\mathcal{L} \in \operatorname{Pic}(A)$ be symmetric. Say $\varphi \in \operatorname{Hom}(B, A)$, by (5) and (6) there are constants $C, C^{\prime}$ which may depend on $\varphi$ such that $\hat{h}_{A, \mathcal{L}}(\varphi(p)) \leq C \hat{h}_{B, \mathcal{M}}(p)+C^{\prime}$ for all $p \in B(\overline{\mathbf{Q}})$. Substituting $p$ by $n p$ for an integer $n$, using the homogeneity of the Néron-Tate height, and taking $n$ to infinity shows that the inequality holds with $C^{\prime}=0$. After choosing a $\mathbf{Z}$-basis of $\operatorname{Hom}(A, B)$ we conclude, from (7) and again from homogeneity of the height, that

$$
\hat{h}_{A, \mathcal{L}}(\varphi(p)) \leq C\|\varphi\|^{2} \hat{h}_{B, \mathcal{M}}(p)
$$

for a new $C$ which is now independent of $\varphi$.

\section{Homomorphisms}

Let $A$ be an abelian variety defined over $\mathbf{C}$. We let $T(A)$ denote the holomorphic tangent space of the Lie group $A(\mathbf{C})$ at the unit element. The association $A \mapsto T(A)$ is functorial: a second abelian variety $B$ and a homomorphism of algebraic groups $\varphi: A \rightarrow B$ give rise to a linear map $T(\varphi): T(A) \rightarrow T(B)$. Furthermore, the identity map on $A$ is taken to the identity map on $T(A)$ and for a third abelian variety $C$ with homomorphism $\psi: B \rightarrow C$ we have $T(\psi \varphi)=T(\psi) T(\varphi)$. For brevity we set

$$
\operatorname{Hom}(A, B)_{\mathbf{Q}}=\operatorname{Hom}(A, B) \otimes_{\mathbf{z}} \mathbf{Q}, \quad \operatorname{Hom}(A, B)_{\mathbf{R}}=\operatorname{Hom}(A, B) \otimes_{\mathbf{Z}} \mathbf{R}
$$

and

$$
\operatorname{End}(A)_{\mathbf{Q}}=\operatorname{End}(A) \otimes_{\mathbf{z}} \mathbf{Q}, \quad \operatorname{End}(A)_{\mathbf{R}}=\operatorname{End}(A) \otimes_{\mathbf{z}} \mathbf{R},
$$

where $\operatorname{End}(A)=\operatorname{Hom}(A, A)$. We will assume $\operatorname{Hom}(A, B) \subset \operatorname{Hom}(A, B)_{\mathbf{Q}} \subset$ $\operatorname{Hom}(A, B)_{\mathbf{R}}$ and $\operatorname{End}(A) \subset \operatorname{End}(A)_{\mathbf{Q}} \subset \operatorname{End}(A)_{\mathbf{R}}$. We obtain an $\mathbf{R}$-linear map

$$
T: \operatorname{Hom}(A, B)_{\mathbf{R}} \rightarrow \operatorname{Hom}(T(A), T(B))
$$

where the right-hand side is the vector space of $\mathbf{C}$-linear maps $T(A) \rightarrow$ $T(B)$. The association stays functorial.

We define

$$
\begin{array}{r}
\operatorname{Hom}(A, B)_{\mathbf{R}}^{*}=\left\{\psi \in \operatorname{Hom}(A, B)_{\mathbf{R}} ;\right. \\
\text { there is } \left.\varphi \in \operatorname{Hom}(B, A)_{\mathbf{R}} \text { with } \psi \varphi=1\right\} .
\end{array}
$$

If $\psi \in \operatorname{Hom}(A, B)_{\mathbf{R}}^{*}$ and $\varphi \in \operatorname{Hom}(B, A)_{\mathbf{R}}^{*}$ with $\psi \varphi=1$, then $T(\psi) T(\varphi)=$ $T(\psi \varphi)=1$. Therefore, the linear map $T(\psi)$ is surjective. Using Poincaré's complete reducibility theorem [16, Corollary 1, p. 174] it is not difficult to show that any surjective homomorphism of algebraic groups $A \rightarrow B$ lies in $\operatorname{Hom}(A, B)_{\mathbf{R}}^{*}$. 
The unit group $\operatorname{End}(B)_{\mathbf{R}}^{*}$ of the ring $\operatorname{End}(B)_{\mathbf{R}}$ acts on $\operatorname{Hom}(A, B)_{\mathbf{R}}^{*}$ by left multiplication. Note that the finite dimensional $\mathbf{R}$-vector space $\operatorname{Hom}(A, B)_{\mathbf{R}}$ carries a natural topology. We will show further down that there exists a compact subspace of $\operatorname{Hom}(A, B)_{\mathbf{R}}^{*}$ whose orbit under this action is $\operatorname{Hom}(A, B)_{\mathbf{R}}^{*}$.

Let $D$ be either $\mathbf{R}, \mathbf{C}$, or the skew-field of quaternions $\mathbf{H}$. For $z \in D$ we define

$$
z^{\prime}= \begin{cases}z & : \text { if } D=\mathbf{R} \\ \bar{z} & : \text { if } D=\mathbf{C}, \\ z^{*}: & \text { if } D=\mathbf{H},\end{cases}
$$

where $z \mapsto \bar{z}$ is complex conjugation and $z \mapsto z^{*}$ is quaternion conjugation. We let $\mathrm{GL}_{n}(D)$ denote the unit group of $\operatorname{Mat}_{n}(D)$, the ring of $n \times n$-matrices with entries in $D$.

Lemma 1. Let $m$ and $n$ be positive integers and let us assume $\psi \in$ $\operatorname{Mat}_{m n}(D)$ has a right inverse in $\operatorname{Mat}_{n m}(D)$. Then $m \leq n$ and there exists $\theta \in \mathrm{GL}_{m}(D)$ and a permutation matrix $\pi \in \mathrm{GL}_{n}(\mathbf{Q})$ such that

$$
\theta \psi \pi=\left[\begin{array}{ccc|ccc}
1 & & & \psi_{11} & \cdots & \psi_{1, n-m} \\
& \ddots & & \vdots & & \vdots \\
& & 1 & \psi_{m 1} & \cdots & \psi_{m, n-m}
\end{array}\right]
$$

and $\psi_{i j} \psi_{i j}^{\prime} \leq 1$ for $1 \leq i \leq m$ and $1 \leq j \leq n-m$.

Proof. By hypothesis, the rows of $\psi$ are left $D$-independent and hence generate an $m$-dimensional left $D$-vector subspace of $D^{n}$; this implies $m \leq n$. We may choose $n-m$ standard basis vectors of $D^{n}$ such that these together with the rows of $\psi$ are a left $D$-basis of $D^{n}$. The matrix whose rows are just these basis vectors lies in $\mathrm{GL}_{n}(D)$. Hence $\psi$ contains an $m \times m$ submatrix which lies in $\mathrm{GL}_{m}(D)$.

We consider $\operatorname{Mat}_{m}(D)$ as a $K$-algebra where $K$ is the center of $\operatorname{Mat}_{m}(D)$. If $D=\mathbf{R}$ or $\mathbf{H}$ then $K=\mathbf{R}$, in the remaining case we have $K=\mathbf{C}$. Let $\theta \in \operatorname{Mat}_{m}(D)$. If $D=\mathbf{R}$ or $\mathbf{C}$, we set $\operatorname{nr}(\theta)=\operatorname{det} \theta$. For $D=\mathbf{H}$ it is wellknown that there is an isomorphism $f: D \otimes_{\mathbf{R}} \mathbf{C} \rightarrow \mathrm{Mat}_{2}(\mathbf{C})$ of $\mathbf{C}$-algebras such that det $f(z \otimes 1)=z z^{\prime}$ for $z \in D$. We may extend $f$ to an isomorphism $f: \operatorname{Mat}_{m}(D) \otimes_{\mathbf{R}} \mathbf{C} \rightarrow \operatorname{Mat}_{2 m}(\mathbf{C})$ and then define $\operatorname{nr}(\theta)=\operatorname{det} f(\theta \otimes 1)$. The map nr: $\operatorname{Mat}_{m}(D) \rightarrow K$ is just the reduced norm. We note that any isomorphism $\operatorname{Mat}_{m}(D) \otimes_{\mathbf{R}} \mathbf{C} \rightarrow \operatorname{Mat}_{2 m}(\mathbf{C})$ leads to the same map. Also, $\operatorname{nr}(\theta)$ is non-zero if and only if $\theta \in \mathrm{GL}_{m}(D)$.

We may find a permutation matrix $\pi \in \mathrm{GL}_{n}(\mathbf{Q})$ such that the modulus of the reduced norm of the $m \times m$ submatrix of $\psi \pi$ determined by the first $m$ columns is maximal among all $m \times m$ submatrices. In particular, this submatrix is in $\mathrm{GL}_{m}(D)$ and we denote it with $\theta^{-1}$. Equation (9) holds.

It remains to prove the bound for $\psi_{i j} \psi_{i j}^{\prime}$. The reduced norm is multiplicative, so by maximality of $\left|\mathrm{nr}\left(\theta^{-1}\right)\right|$ the absolute value of the reduced 
norm of

$$
\left[\begin{array}{ccccc}
1 & & \psi_{1 j} & & \\
& \ddots & & & \\
& & \vdots & & \\
& & & \ddots & \\
& & \psi_{m j} & & 1
\end{array}\right]
$$

is at most 1 ; here the $i$ th column of the identity matrix has been replaced by the transpose of $\left(\psi_{1 j}, \ldots, \psi_{m j}\right)$. If $K=\mathbf{R}$ or $\mathbf{C}$, the reduced norm of the matrix (10) equals its determinant $\psi_{i j}$. Hence $\left|\psi_{i j}\right| \leq 1$, which implies $\psi_{i j} \psi_{i j}^{\prime} \leq 1$. If $K=\mathbf{H}$ and using $f$ as above, the reduced norm of (10) equals $\psi_{i j} \psi_{i j}^{\prime} \leq 1$.

Based on this lemma we now show a compactness property of left-action of $\operatorname{End}(B)_{\mathbf{R}}^{*}$ on $\operatorname{Hom}(A, B)_{\mathbf{R}}^{*}$.

Lemma 2. There exists a compact subspace $\mathcal{K} \subset \operatorname{Hom}(A, B)_{\mathbf{R}}^{*}$ with the following property: if $\psi \in \operatorname{Hom}(A, B)_{\mathbf{R}}^{*}$ there is $\theta \in \operatorname{End}(B)_{\mathbf{R}}$ with $\theta \psi \in \mathcal{K}$.

Proof. By Poincaré's complete reducibility theorem $A$ is isogenous to $A_{1}^{n_{1}} \times$ $\cdots \times A_{l}^{n_{l}}$ and $B$ is isogenous to $A_{1}^{m_{1}} \times \cdots \times A_{l}^{m_{l}}$ where the $A_{i}$ are simple, pairwise non-isogenous abelian varieties defined over $\mathbf{C}$ and $n_{i}, m_{i}$ are integers not both zero. After tensoring with $\mathbf{R}$ we see

$$
\operatorname{Hom}(A, B)_{\mathbf{R}}=\bigoplus_{i=1}^{l} \operatorname{Hom}\left(A_{i}^{n_{i}}, A_{i}^{m_{i}}\right)_{\mathbf{R}} .
$$

Since $\operatorname{Hom}\left(A_{i}^{n_{i}}, A_{i}^{m_{i}}\right)_{\mathbf{R}}=\operatorname{Mat}_{m_{i} n_{i}}\left(\operatorname{End}\left(A_{i}\right)_{\mathbf{R}}\right)$ we have

$$
\operatorname{Hom}(A, B)_{\mathbf{R}}=\bigoplus_{i=1}^{l} \operatorname{Mat}_{m_{i} n_{i}}\left(\operatorname{End}\left(A_{i}\right)_{\mathbf{R}}\right)
$$

By [16, Theorem 2, p. 201] the ring $\operatorname{End}\left(A_{i}\right)_{\mathbf{R}}$ is the direct sum of matrix rings with coefficients either $\mathbf{R}, \mathbf{C}$, or $\mathbf{H}$. In order to prove the lemma it suffices to show the following statement:

Let $m$ and $n$ be positive integers and let $D=\mathbf{R}, \mathbf{C}$, or $\mathbf{H}$. There exists a compact subspace

$$
\mathcal{K} \subset\left\{\psi \in \operatorname{Mat}_{m n}(D) ; \psi \text { has a right inverse in } \operatorname{Mat}_{n m}(D)\right\}
$$

such that for any $\psi \in \operatorname{Mat}_{m n}(D)$ with right inverse in $\operatorname{Mat}_{n m}(D)$ there is $\theta \in \mathrm{GL}_{m}(D)$ with $\theta \psi \in \mathcal{K}$.

We apply Lemma 1 to prove this statement. The set $\mathcal{K}^{\prime}$ of matrices as on the right-hand side of (9) subject to $\psi_{i j} \psi_{i j}^{\prime} \leq 1$ is clearly contained in (11). Furthermore, it is also compact since it is a closed and bounded 
subset of a real vector space of finite dimension. We now define $\mathcal{K}$ to be the union $\bigcup_{\pi} \mathcal{K}^{\prime} \pi$ where $\pi$ runs over the finite set of permutation matrices in $\operatorname{Mat}_{n}(\mathbf{Q})$. The statement below (11) follows from Lemma 1.

In fact $\theta$ even lies in $\operatorname{End}(B)_{\mathbf{R}}^{*}$, but we will not use this property directly.

\section{Auxiliary morphism and an upper bound}

In this section $A$ and $B$ denote abelian varieties defined over $\overline{\mathbf{Q}}$. We suppose that $\mathcal{L}_{A}, \mathcal{L}_{B}$ are symmetric ample line bundles on $A, B$ respectively. For brevity we write $\hat{h}_{A}, \hat{h}_{B}$ instead of $\hat{h}_{A, \mathscr{L}_{A}}, \hat{h}_{B, \mathscr{L}_{B}}$ respectively.

If $\pi_{1}: A \times B \rightarrow A$ and $\pi_{2}: A \times B \rightarrow B$ are the natural projections, then $\pi_{1}^{*} \mathcal{L}_{A} \otimes \pi_{2}^{*} \mathcal{L}_{B}$ is an ample line bundle on $A \times B$ and as such defines a polarization. This polarization induces, by virtue of the Rosati involution, a norm on $\operatorname{End}(A \times B)_{\mathbf{R}}$. There is a natural injection $\operatorname{Hom}(A, B) \rightarrow \operatorname{End}(A \times B)$ defined by sending $\varphi \in \operatorname{Hom}(A, B)$ to the morphism $(p, q) \mapsto(0, \varphi(p))$. This map extends to an injection $\operatorname{Hom}(A, B)_{\mathbf{R}} \rightarrow \operatorname{End}(A \times B)_{\mathbf{R}}$. Therefore, the norm defined above on $\operatorname{End}(A \times B)_{\mathbf{R}}$ restricts to a norm $\|\cdot\|$ on $\operatorname{Hom}(A, B)_{\mathbf{R}}$. For the sake of definiteness we will work with this norm through the article, although any choice of norm on $\operatorname{Hom}(A, B)_{\mathbf{R}}$ is apt. This construction also works for abelian varieties over defined $\mathbf{C}$.

The following result on simultaneous approximation of real numbers is a well known application of the box principle.

Lemma 3. Let $\xi_{1}, \ldots, \xi_{\rho} \in \mathbf{R}$ and let $Q>1$, there exist integers $q, q_{1}$, $\ldots, q_{\rho}$ with $1 \leq q \leq Q$ and

$$
\left|q \xi_{i}-q_{i}\right|<Q^{-1 / \rho} \quad \text { for } 1 \leq i \leq \rho .
$$

Proof. This follows from [9, Theorem VI, p. 13].

Recall that $\operatorname{Hom}(A, B)$ is a finitely generated free $\mathbf{Z}$-module. We let $\rho=\operatorname{rk} \operatorname{Hom}(A, B)$ where rk is the rank of any finitely generated $\mathbf{Z}$-module. To avoid trivialities we shall assume $\rho>0$ throughout this section.

Lemma 4. There is a positive constant $C_{1}$ depending on $A, B, \mathscr{L}_{A}$, and $\mathscr{L}_{B}$ with the following property: if $Q>1$ and if $\varphi_{0} \in \operatorname{Hom}(A, B)_{\mathbf{R}}$ there exist $q \in \mathbf{Z}$ and $\varphi \in \operatorname{Hom}(A, B)$ such that

$$
1 \leq q \leq Q \quad \text { and } \quad\left\|q \varphi_{0}-\varphi\right\| \leq C_{1} Q^{-1 / \rho} .
$$

Proof. Let us fix a Z Z-basis $\varphi_{1}, \ldots, \varphi_{\rho}$ of $\operatorname{Hom}(A, B)$. Now $\varphi_{0}=\xi_{1} \varphi_{1}+$ $\cdots+\xi_{\rho} \varphi_{\rho}$ for some $\xi_{i} \in \mathbf{R}$ and we use the previous lemma to approximate $\xi_{i}$ by $q_{i} / q$. The lemma then follows with $\varphi=q_{1} \varphi_{1}+\cdots+q_{\rho} \varphi_{\rho}$ by applying the triangle inequality.

The compact subspace $\mathcal{K} \subset \operatorname{Hom}(A, B)_{\mathbf{R}}^{*}$ given by Lemma 2 is used in the next lemma. 
Lemma 5. There are positive constants $C_{3}, C_{4}$ depending on $A, B, \mathscr{L}_{A}, \mathscr{L}_{B}$, and $\mathcal{K}$ with the following property: if $Q>1$ and if $p \in A(\overline{\mathbf{Q}})$ vanishes at an element of $\operatorname{Hom}(A, B) \cap \operatorname{Hom}(A, B)_{\mathbf{R}}^{*}$ then there exist $q \in \mathbf{Z}$, $\varphi \in \operatorname{Hom}(A, B)$, and $\varphi_{0} \in \mathcal{K}$ such that

$1 \leq q \leq Q, \quad \hat{h}_{B}(\varphi(p)) \leq C_{3} Q^{-2 / \rho} \hat{h}_{A}(p), \quad$ and $\quad\left\|q \varphi_{0}-\varphi\right\| \leq C_{4} Q^{-1 / \rho}$.

Proof. Say $\psi \in \operatorname{Hom}(A, B) \cap \operatorname{Hom}(A, B)_{\mathbf{R}}^{*}$ vanishes at $p$. By Lemma 2 we may find $\theta \in \operatorname{End}(B)_{\mathbf{R}}$ such that $\varphi_{0}=\theta \psi \in \mathcal{K}$. It will prove more adequate to work with an approximation of $\theta$ lying in $\operatorname{End}(B)_{\mathbf{Q}}$. Since this set lies dense in $\operatorname{End}(B)_{\mathbf{R}}$ we may find $\tilde{\theta} \in \operatorname{End}(B)_{\mathbf{Q}}$ with

$$
\|\theta \psi-\tilde{\theta} \psi\| \leq C_{1} Q^{-1-1 / \rho}
$$

where $C_{1}$ is from Lemma 4 . We apply this lemma to $\tilde{\theta} \psi$ to find $1 \leq q \leq Q$ and $\varphi \in \operatorname{Hom}(A, B)$ with

$$
\|\delta\| \leq C_{1} Q^{-1 / \rho} \quad \text { where } \delta=q \tilde{\theta} \psi-\varphi .
$$

Let $N$ be a positive integer with $N \tilde{\theta} \in \operatorname{End}(B)$, then $N \delta \in \operatorname{Hom}(A, B)$ and

$$
(N \delta)(p)=q(N \tilde{\theta})(\psi(p))-N \varphi(p)=-N \varphi(p)
$$

because $\psi(p)=0$. Certainly, the above expression does not make sense with $\tilde{\theta}$ replaced by $\theta$, since the latter only lies in $\operatorname{End}(B)_{\mathbf{R}}$.

The height is a quadratic form, so $N^{2} \hat{h}_{B}(\varphi(p))=\hat{h}_{B}(-N \varphi(p))=$ $\hat{h}_{B}((N \delta)(p))$. Furthermore, by (8) we can bound the height of $(N \delta)(p)$ from above by $C_{2}\|N \delta\|^{2} \hat{h}_{A}(p)$ with $C_{2}$ independent of $p$ and $N \delta$. The approximation inequality (14) implies $N^{2} \hat{h}_{B}(\varphi(p)) \leq C_{1}^{2} C_{2} N^{2} Q^{-2 / \rho} \hat{h}_{A}(p)$. The middle inequality in (12) follows with $C_{3}=C_{1}^{2} C_{2}$ on cancelling $N^{2}$.

The last inequality in (12) follows with $C_{4}=2 C_{1}$ from

$$
\begin{aligned}
\left\|q \varphi_{0}-\varphi\right\| & =\|q \theta \psi-\varphi-q \tilde{\theta} \psi+q \tilde{\theta} \psi\| \leq q\|\theta \psi-\tilde{\theta} \psi\|+\|q \tilde{\theta} \psi-\varphi\| \\
& \leq C_{1} q Q^{-1-1 / \rho}+\|\delta\| \leq 2 C_{1} Q^{-1 / \rho}
\end{aligned}
$$

where we used (13), $q \leq Q$, and (14).

\section{A lower bound}

Let $A$ and $B$ be abelian varieties defined over $\mathbf{C}$ and let $\mathcal{L}_{A}$ and $\mathcal{L}_{B}$ be symmetric ample line bundles on $A, B$ respectively. We assume $\operatorname{Hom}(A, B) \neq 0$ to avoid trivialities. Let $\|\cdot\|$ be the norm on $\operatorname{Hom}(A, B)_{\mathbf{R}}$ chosen in Sect. 4. Furthermore, $X$ will denote an irreducible closed subvariety of $A$ of dimension $r \geq 1$ defined over $\mathbf{C}$.

For $\varphi \in \operatorname{Hom}(A, B)$ and for an integer $k$ with $0 \leq k \leq r$, we set

$$
v_{X}^{k}(\varphi)=\left(c_{1}\left(\left.\varphi\right|_{X} ^{*} \mathcal{L}_{B}\right)^{k} c_{1}\left(\left.\mathscr{L}_{A}\right|_{X}\right)^{r-k}[X]\right) ;
$$


here $c_{1}$ takes a line bundle to its first Chern class, $[X]$ is the element in the group of cycles on $A$ modulo rational equivalence corresponding to $X$, and $(\cdot)$ denotes the degree of the class of a cycle on $A$ (cf. [11, Chaps. 1 and 2]). Certainly, $v_{X}^{k}(\varphi)$ depends on the choice of the pair $\mathcal{L}_{A}, \mathcal{L}_{B}$ but only on $\mathcal{L}_{B}$ in the important case $k=r$. We abbreviate

$$
v_{X}(\varphi)=v_{X}^{r}(\varphi)
$$

For us, the only relevant cases will be $k=r$ and $k=r-1$.

We begin by showing how to extend $v_{X}^{k}$ to a continuous map $\operatorname{Hom}(A, B)_{\mathbf{R}} \rightarrow \mathbf{R}$.

Let $R$ be a ring and $M$ and $N$ two $R$-modules. A map $f: M \rightarrow N$ is called polynomial if it can be written as a finite sum of terms $f_{1} \cdots f_{d} n$ where the $f_{i}: M \rightarrow R$ are $R$-linear and $n \in N$. Moreover, we call $f$ homogeneous of degree $d$ if in each term the number of $R$-linear factors is $d$.

We recall that $\operatorname{Hom}(A, B)$ is a finitely generated and free $\mathbf{Z}$-module. It follows from the theorem of the cube [16, Corollary 2, pp. 58-59] and since $\mathcal{L}_{B}$ is symmetric that $\left.\varphi \mapsto \varphi\right|_{X} ^{*} \mathcal{L}_{B}$ is a polynomial map $\operatorname{Hom}(A, B) \rightarrow$ $\operatorname{Pic}(X)$ (with $R=\mathbf{Z}$ ) which is homogeneous of degree 2. From basic properties of the degree of cycle classes and the definition we conclude that $\varphi \mapsto v_{X}^{k}(\varphi)$ is a polynomial map $\operatorname{Hom}(A, B) \rightarrow \mathbf{Z}$ which is homogeneous of degree $2 k$. Hence

$$
v_{X}^{k}(\lambda \varphi)=\lambda^{2 k} v_{X}^{k}(\varphi) \quad \text { for } \lambda \in \mathbf{Z} \text { and } \varphi \in \operatorname{Hom}(A, B) .
$$

Now $v_{X}^{k}$ can be extended to a polynomial map $v_{X}^{k}: \operatorname{Hom}(A, B)_{\mathbf{R}} \rightarrow \mathbf{R}$ (with $R=\mathbf{R}$ ) which remains homogeneous of degree $2 k$. In particular, $v_{X}^{k}$ is continuous on the finite dimensional $\mathbf{R}$-vector space $\operatorname{Hom}(A, B)_{\mathbf{R}}$.

Lemma 6. The polynomial map $v_{X}^{k}$ satisfies the following properties.

(i) There is a positive constant $C_{5}$ depending on $X, A, B, \mathcal{L}_{A}$, and $\mathcal{L}_{B}$ such that for all $\varphi \in \operatorname{Hom}(A, B)_{\mathbf{R}}$ we have

$$
v_{X}^{k}(\varphi) \leq C_{5}\|\varphi\|^{2 k}
$$

(ii) For all $p \in A(\mathbf{C})$ and $\varphi \in \operatorname{Hom}(A, B)_{\mathbf{R}}$ we have

$$
v_{p+X}(\varphi)=v_{X}(\varphi)
$$

In other words $v_{X}$ is invariant under translating $X$.

(iii) For all $\varphi \in \operatorname{Hom}(A, B)$ we have

$$
v_{X}(\varphi)=\operatorname{deg}\left(\left.\varphi\right|_{X}\right)\left(c_{1}\left(\left.\mathcal{L}_{B}\right|_{\varphi(X)}\right)^{r}[\varphi(X)]\right),
$$

here $\operatorname{deg}\left(\left.\varphi\right|_{X}\right)$ is the degree of the morphism $\left.\varphi\right|_{X}: X \rightarrow \varphi(X)$.

(iv) For all $\varphi \in \operatorname{Hom}(A, B)_{\mathbf{R}}$ we have $v_{X}(\varphi) \geq 0$. 
Proof. Part (i) follows immediately from the fact that $v_{X}^{k}$ is polynomial and homogeneous of degree $2 k$ on the finite dimensional $\mathbf{R}$-vector space $\operatorname{Hom}(A, B)_{\mathbf{R}}$.

To show part (ii) it suffices to show the equality for $\varphi \in \operatorname{Hom}(A, B)$. Let $t_{p}: A \rightarrow A$ denote the translation by $p \in A(\mathbf{C})$ morphism. Then $t_{p_{*}}[X]=[p+X]$ and so by the projection formula

$$
\begin{aligned}
v_{p+X}(\varphi) & =\left(c_{1}\left(\left.\varphi\right|_{p+X} ^{*} \mathcal{L}_{B}\right)^{r}[p+X]\right)=\left(c_{1}\left(\varphi^{*} \mathcal{L}_{B}\right)^{r}[p+X]\right) \\
& =\left(c_{1}\left(\varphi^{*} \mathcal{L}_{B}\right)^{r} t_{p_{*}}[X]\right)=\left(c_{1}\left(t_{p}{ }^{*} \varphi^{*} \mathcal{L}_{B}\right)^{r}[X]\right) .
\end{aligned}
$$

It is well-known that $t_{p}{ }^{*} \mathcal{M} \otimes \mathcal{M}^{\otimes(-1)}$ is algebraically equivalent to zero for all $\mathcal{M} \in \operatorname{Pic}(A)$. With $\mathcal{M}=\varphi^{*} \mathscr{L}_{B}$ we get

$$
v_{p+X}(\varphi)=\left(c_{1}\left(\varphi^{*} \mathcal{L}_{B}\right)^{r}[X]\right)=v_{X}(\varphi) .
$$

Part (iii) follows directly from the projection formula.

To show part (iv) we note that part (iii) and homogeneity imply that $v$ is non-negative on $\operatorname{Hom}(A, B)_{\mathbf{Q}}$. By continuity we conclude the same on $\operatorname{Hom}(A, B)_{\mathbf{R}}$.

For the remainder of this section we suppose that all varieties are defined over $\overline{\mathbf{Q}}$. For brevity we set $\hat{h}_{A, \mathscr{L}_{A}}=\hat{h}_{A}$ and $\hat{h}_{B, \mathscr{L}_{B}}=\hat{h}_{B}$.

The next lemma uses a criterion of Siu [15, Theorem 2.2.15, p. 143], to prove the existence of a non-zero global section of a certain line bundle. This global section will lead to a height lower bound. For the reader's convenience we briefly recall Siu's criterion. A line bundle $\mathcal{L}$ on an irreducible projective variety is called numerically effective, or nef, if $\left(c_{1}(\mathcal{L})[C]\right) \geq 0$ for every irreducible curve $C$ on said variety. Examples of nef line bundles are ample line bundles, non-negative powers of nef line bundles, and pull-backs of nef line bundles. Siu's theorem states that if $\mathcal{L}$ and $\mathcal{M}$ are nef line bundles on $X$ satisfying $\left(c_{1}(\mathcal{L})^{r}[X]\right)>r\left(c_{1}(\mathcal{L})^{r-1} c_{1}(\mathcal{M})[X]\right)$, then there exists a positive integer $k$ such that $\left(\mathcal{L} \otimes \mathcal{M}^{\otimes(-1)}\right)^{\otimes k}$ admits a non-zero global section. The numerical constant $r$ which appears in the inequality of intersection numbers is not relevant for our work, in fact our method works with any constant which depends only on $X$.

Lemma 7. There exists a positive constant $C_{8}$ depending on $A, B, \mathcal{L}_{A}$, and $\mathcal{L}_{B}$ with the following property: let $\varphi \in \operatorname{Hom}(A, B)$ be non-zero, there is a Zariski open dense subset $U \subset X$ and $C_{7} \in \mathbf{R}$ such that if $p \in U(\overline{\mathbf{Q}})$ then

$$
\hat{h}_{B}(\varphi(p)) \geq C_{8}\|\varphi\|^{2} \frac{v_{X}(\varphi)}{\|\varphi\|^{2 r}} \hat{h}_{A}(p)-C_{7},
$$

here $C_{7}$ may depend on $\varphi$ but it does not depend on $p$.

Proof. If $v_{X}(\varphi)=0$, then the lemma follows since $\hat{h}_{B}(\varphi(x))=\hat{h}_{B, \mathscr{L}_{B}}(\varphi(x))$ $\geq 0$ by ampleness of $\mathcal{L}_{B}$. Therefore we may assume $v_{X}(\varphi) \neq 0$ and hence $v_{X}(\varphi)>0$ by Lemma 6(iv). 
We fix positive integers $m=v_{X}(\varphi)$ and $n=2 r \max \left\{1, v_{X}^{r-1}(\varphi)\right\}$, so

$$
\frac{v_{X}(\varphi)}{2 r \max \left\{1, v_{X}^{r-1}(\varphi)\right\}}=\frac{m}{n}<\frac{v_{X}(\varphi)}{r \max \left\{1, v_{X}^{r-1}(\varphi)\right\}} .
$$

By multiplying the inequality above with $n^{r}$ we obtain

$$
n^{r} v_{X}(\varphi)>n^{r-1} m r v_{X}^{r-1}(\varphi) .
$$

Additivity properties of the degree give

$$
\begin{aligned}
\left(c_{1}\left(\left.\varphi\right|_{X} ^{*} \mathcal{L}_{B}^{\otimes n}\right)^{r}[X]\right) & =n^{r} v_{X}(\varphi) \\
& >n^{r-1} m r v_{X}^{r-1}(\varphi)=r\left(c_{1}\left(\left.\varphi\right|_{X} ^{*} \mathcal{L}_{B}^{\otimes n}\right)^{r-1} c_{1}\left(\left.\mathcal{L}_{A}^{\otimes m}\right|_{X}\right)[X]\right) .
\end{aligned}
$$

By Siu's theorem we conclude that a positive power of

$$
\left.\left.\varphi\right|_{X} ^{*} \mathcal{L}_{B}^{\otimes n} \otimes\left(\mathcal{L}_{A}^{\otimes m}\right)\right|_{X} ^{\otimes(-1)}=\left.\left(\varphi^{*} \mathcal{L}_{B}^{\otimes n} \otimes \mathcal{L}_{A}^{\otimes(-m)}\right)\right|_{X}
$$

has a non-zero global section. By (3) and the comment following this statement, there exists a Zariski open and dense $U \subset X$ such that the height on $X$ associated to (19) is bounded from below on $U(\overline{\mathbf{Q}})$. By (6) and functional properties, the Néron-Tate height on $A(\overline{\mathbf{Q}})$ associated to $\varphi^{*} \mathcal{L}_{B}^{\otimes n} \otimes \mathcal{L}_{A}^{\otimes(-m)}$ is bounded from below on $U(\overline{\mathbf{Q}})$ by $-C_{6}$. Using again the functional properties we obtain

$$
\begin{aligned}
-C_{6} \leq \hat{h}_{A, \varphi^{*} \mathcal{L}_{B}^{\otimes n} \otimes \mathcal{L}_{A}^{\otimes(-m)}}(p) & =\hat{h}_{A, \varphi^{*} \mathcal{L}_{B}^{\otimes n}}(p)-\hat{h}_{A, \mathcal{L}_{A}^{\otimes m}}(p) \\
& =n \hat{h}_{B}(\varphi(p))-m \hat{h}_{A}(p)
\end{aligned}
$$

for $p \in U(\overline{\mathbf{Q}})$; we note that $C_{6}$ is independent of $p$. We apply the equality in (18) to deduce

$$
\hat{h}_{B}(\varphi(p)) \geq \frac{v_{X}(\varphi)}{2 r \max \left\{1, v_{X}^{r-1}(\varphi)\right\}} \hat{h}_{A}(p)-C_{7}
$$

for all $p \in U(\overline{\mathbf{Q}})$ with $C_{7}$ independent of $p$.

The upper bound for $v_{X}^{r-1}(\varphi)$ given in Lemma 6(i) combined with (20) leads to

$$
\hat{h}_{B}(\varphi(p)) \geq \frac{v_{X}(\varphi)}{2 r \max \left\{1, C_{5}\|\varphi\|^{2(r-1)}\right\}} \hat{h}_{A}(p)-C_{7} .
$$

Finally, since $\varphi$ is contained in the discrete subgroup $\operatorname{Hom}(A, B)$ of $\operatorname{Hom}(A, B)_{\mathbf{R}}$ and $\varphi \neq 0$ we may suppose that $C_{5}\|\varphi\|^{2(r-1)} \geq 1$ after replaced $C_{5}$ by a larger constant if necessary. Inequality (17) follows. 
In proving the bounded height theorem it is natural to consider only those $X$ which satisfy

$$
v_{X}(\varphi)>0 \quad \text { for all } \varphi \in \operatorname{Hom}(A, B) \cap \operatorname{Hom}(A, B)_{\mathbf{R}}^{*}
$$

for all $B$ of dimension at least $s$, since otherwise we have $X^{\mathrm{oa},[s]}=\emptyset$. Indeed, say $\varphi \in \operatorname{Hom}(A, B) \cap \operatorname{Hom}(A, B)_{\mathbf{R}}^{*}$ with $v_{X}(\varphi)=0$. Now $\varphi$ : $A \rightarrow B$ is a surjective homomorphism and $\operatorname{deg}\left(\left.\varphi\right|_{X}\right)=0$ by (16). Therefore, $\operatorname{dim} \varphi(X)<\operatorname{dim} X=r$. Let $p \in X(\mathbf{C})$, by the theorem on the dimension of the fibres [10, first theorem on p. 228] we conclude that $\left.\varphi\right|_{X} ^{-1}(\varphi(p))$ has positive dimension. But the irreducible components of this Zariski closed set are contained in the translate of the kernel of $\varphi$ which itself is an algebraic group of dimension $\operatorname{dim} A-\operatorname{dim} B$. From the definition we deduce that $p \notin X^{\mathrm{oa},[s]}$. Since $p$ was arbitrary we obtain $X^{\mathrm{oa},[s]}=\emptyset$.

In Proposition 1 we prove a weak version of the main result under the apparently stronger non-vanishing hypothesis

$$
v_{X}(\varphi)>0 \quad \text { for all } \varphi \in \operatorname{Hom}(A, B)_{\mathbf{R}}^{*} .
$$

In this case $v_{X}$ is bounded below by a positive constant on any compact subspace of $\operatorname{Hom}(A, B)_{\mathbf{R}}^{*}$, in particular on $\mathcal{K}$ from Lemma 2 .

Hypothesis (21) will be studied in more detail for $A$ and $B$ both powers of an elliptic curve in Sect. 6 and for general $A$ and $B$ in Sect. 8.

For the proposition we need the following simple observation: let $p=$ $a+b$ where $a, b \in A(\overline{\mathbf{Q}}), \hat{h}_{A}(b) \leq \epsilon\left(1+\hat{h}_{A}(a)\right)$ and $0 \leq \epsilon \leq 1 / 3$. By (7) we have $\hat{h}_{A}(a)=\hat{h}_{A}(p-b) \leq 2 \hat{h}_{A}(p)+2 \hat{h}_{A}(b)$ and so $\hat{h}_{A}(a) \leq$ $2 \hat{h}_{A}(p)+2 \epsilon\left(1+\hat{h}_{A}(a)\right) \leq 2 \hat{h}_{A}(p)+2\left(1+\hat{h}_{A}(a)\right) / 3$. Hence

$$
\hat{h}_{A}(a) \leq 2+6 \hat{h}_{A}(p) \leq 6\left(1+\hat{h}_{A}(p)\right) \quad \text { and } \quad \hat{h}_{A}(b) \leq 6 \epsilon\left(1+\hat{h}_{A}(p)\right) .
$$

Proposition 1. Assume that $X$ satisfies hypothesis (21). There exist a Zariski open dense $U \subset X$, an $\epsilon>0$, and a constant $C_{19}$ with the following property: if $p=a+b \in U(\overline{\mathbf{Q}})$ such that

(1) $a, b \in A(\overline{\mathbf{Q}})$,

(2) there exists a surjective $\psi \in \operatorname{Hom}(A, B)$ with $\psi(a)=0$, and

(3) $\hat{h}_{A}(b) \leq \epsilon\left(1+\hat{h}_{A}(a)\right)$

then $\hat{h}_{A}(p) \leq C_{19}$.

Proof. Let $C_{9}, \ldots, C_{18}$ denote positive constants which depend on $X, A, B$ etc. but not on $p$.

Let $\mathcal{K}$ be the compact subspace of $\operatorname{Hom}(A, B)_{\mathbf{R}}$ from Lemma 2 and let $C_{9}$ be an upper bound for $\|\cdot\|$ on $\mathcal{K}$.

Recall that $v_{X}$ is continuous on $\operatorname{Hom}(A, B)_{\mathbf{R}}$ and because of (21) it does not vanish on $\mathcal{K}$. By compactness $v_{X}$ is bounded below on $\mathcal{K}$ by a positive 
constant. We may even conclude that there exists $\kappa>0$ and $C_{10}>0$ such that

$$
\begin{array}{r}
\text { if } \varphi_{0}^{\prime} \in \operatorname{Hom}(A, B)_{\mathbf{R}} \text { and } \varphi_{0} \in \mathcal{K} \text { with }\left\|\varphi_{0}^{\prime}-\varphi_{0}\right\| \leq \kappa \\
\text { then } v_{X}\left(\varphi_{0}^{\prime}\right) \geq C_{10}>0 .
\end{array}
$$

We assume that $Q>1$ is large with respect to $\kappa$ and the $C_{i}$; we also assume that $\epsilon \in(0,1 / 3]$ is small with respect to $Q$. The two shall not depend on $p, a, b, \psi$ and we will define them properly further down.

Let $\rho$ be the rank of $\operatorname{Hom}(A, B)$ as a free $\mathbf{Z}$-module, recall that $\rho>0$. We define $\Phi$ to be the set of $\varphi \in \operatorname{Hom}(A, B)$ such that there exist an integer $q$ and $\varphi_{0} \in \mathcal{K}$ with $1 \leq q \leq Q$ and $\left\|q \varphi_{0}-\varphi\right\| \leq C_{4} Q^{-1 / \rho}$. Any $\varphi$ provided by Lemma 5 lies in $\Phi$. Moreover,

$$
\|\varphi\| \leq q\left\|\varphi_{0}\right\|+C_{4} Q^{-1 / \rho} \leq C_{9} q+C_{4} Q^{-1 / \rho} \leq C_{11} q,
$$

so the set $\Phi \subset \operatorname{Hom}(A, B)$ is bounded and hence finite. We may assume

$$
C_{4} Q^{-1 / \rho} \leq \kappa .
$$

Using this inequality we see that $\varphi_{0}^{\prime}=\varphi / q$ and $\varphi_{0}$ satisfy (23), so $v_{X}(\varphi / q) \geq$ $C_{10}>0$. In particular, we have $\varphi \neq 0$, and so $0 \notin \Phi$. By homogeneity (15) we get

$$
\frac{v_{X}(\varphi)}{q^{2 r}} \geq C_{10}
$$

and the bound (24) gives

$$
\frac{v_{X}(\varphi)}{\|\varphi\|^{2 r}} \geq \frac{C_{10}}{C_{11}^{2 r}}
$$

For each $\varphi \in \Phi$ we get a Zariski open non-empty set as in Lemma 7; let $U$ be their intersection as $\varphi$ runs over all elements of $\Phi$. Then $U$ is Zariski open and non-empty since $\Phi$ is finite. Moreover, there is a constant $C(Q)$ such that for all $\varphi \in \Phi$ and all $p \in U(\overline{\mathbf{Q}})$ we have

$$
\hat{h}_{B}(\varphi(p)) \geq C_{6}\|\varphi\|^{2} \frac{v_{X}(\varphi)}{\|\varphi\|^{2 r}} \hat{h}_{A}(p)-C(Q) .
$$

Together with the lower bound from (25) we obtain

$$
\hat{h}_{B}(\varphi(p)) \geq C_{12}\|\varphi\|^{2} \hat{h}_{A}(p)-C(Q) .
$$

Finally, since $\operatorname{Hom}(A, B)$ is discrete and since $\varphi \neq 0$ we have $\|\varphi\|^{2} \geq C_{13}$ and therefore

$$
\hat{h}_{B}(\varphi(p)) \geq C_{14} \hat{h}_{A}(p)-C(Q) .
$$

The point is that this inequality holds for all $\varphi \in \Phi$ and all $p \in U(\overline{\mathbf{Q}})$. 
Let us assume that $p \in U(\overline{\mathbf{Q}}), a, b$, and $\psi$ are as in the hypothesis. By Lemma 5 there exists $\varphi \in \Phi$ with $\hat{h}_{B}(\varphi(a)) \leq C_{3} Q^{-2 / \rho} \hat{h}_{A}(a)$. By (22) the height $\hat{h}_{A}(a)$ is not much larger than $\hat{h}_{A}(p)$, more precisely

$$
\hat{h}_{B}(\varphi(a)) \leq 6 C_{3} Q^{-2 / \rho}\left(1+\hat{h}_{A}(p)\right) .
$$

We can bound the height of $\varphi(b)$ from above using $(8)$ and get $\hat{h}_{B}(\varphi(b)) \leq$ $C_{15}\|\varphi\|^{2} \hat{h}_{A}(b)$. By (24) and $q \leq Q$ the norm $\|\varphi\|$ is bounded in terms of $Q$ yielding $\hat{h}_{B}(\varphi(b)) \leq C_{16} Q^{2} \hat{h}_{A}(b)$. Finally, by $(22) \hat{h}_{A}(b)$ is small compared to $\hat{h}_{A}(p)$, hence

$$
\hat{h}_{B}(\varphi(b)) \leq C_{17} \epsilon Q^{2}\left(1+\hat{h}_{A}(p)\right) .
$$

Now $\varphi(p)=\varphi(a)+\varphi(b)$ so by (7), (27), and (28) we get

$$
\hat{h}_{B}(\varphi(p)) \leq C_{18}\left(Q^{-2 / \rho}+\epsilon Q^{2}\right)\left(1+\hat{h}_{A}(p)\right) .
$$

We may assume that $Q$ is fixed with $C_{18} Q^{-2 / \rho} \leq C_{14} / 3$. Then let us set

$$
\epsilon=\min \left\{\frac{1}{3}, \frac{C_{14}}{3 C_{18} Q^{2}}\right\} \in(0,1 / 3]
$$

Inequality (29) implies

$$
\hat{h}_{B}(\varphi(p)) \leq \frac{2}{3} C_{14}\left(1+\hat{h}_{A}(p)\right)
$$

and the proof follows on comparing this upper bound with the lower bound in (26).

By Poincaré's complete reducibility theorem we can choose finitely many abelian varieties $B_{1}, \ldots, B_{n}$ of dimension at least $\operatorname{dim} X$ with the following property: if $p \in A^{[\operatorname{dim} X]}$ there is a surjective homomorphism $\psi: A \rightarrow B_{i}$ with $\psi(p)=0$ for some $i$. This $\psi$ lies in $\operatorname{Hom}\left(A, B_{i}\right)_{\mathbf{R}}^{*}$ since it is surjective. If $X$ satisfies (21) for all $B_{1}, \ldots, B_{n}$, then the previous Proposition shows that there exists $U \subset X$ Zariski open and dense such that the Néron-Tate height is bounded on $U(\overline{\mathbf{Q}}) \cap A^{[\mathrm{dim} X]}$. This gives a weak variant of the bounded height theorem in the critical case $s=\operatorname{dim} X$.

\section{Subvarieties of a power of an elliptic curve}

We will see that the proof of the bounded height theorem involves a careful study of the non-vanishing hypothesis (21). As a warm-up we demonstrate a weaker version for a power of an elliptic curve without complex multiplication.

Proposition 2. Let $E$ be an elliptic curve without complex multiplication and let $X$ be an irreducible closed subvariety of $E^{g}$ both defined over $\overline{\mathbf{Q}}$. Suppose $X^{\mathrm{oa}} \neq \emptyset$, then there exists a Zariski open and non-empty $U \subset X$ 
such that the Néron-Tate height associated to an ample symmetric line bundle on $E^{g}$ is bounded on $U(\overline{\mathbf{Q}}) \cap\left(E^{g}\right)^{[\operatorname{dim} X]}$.

Many steps of the proof of the main result show up in simplified form in the proof of this proposition. For the sake of clarity we provide a proof. And in order to keep the exposition simple we have added the non-complex multiplication hypothesis on $E$. The proof of bounded height theorem itself does not use Proposition 2.

Let $E$ be any elliptic curve defined over C. Let $p \in\left(E^{g}\right)^{[r]}$ and say $H$ is an algebraic subgroup of codimension at least $r$ containing $p$. Then there exist an abelian variety $B$ of dimension at least $r$ and a surjective morphism $\psi: E^{g} \rightarrow B$ such that $H$ has finite index in the kernel of $\psi$. By the theorem on the dimension of the fibres, $\operatorname{dim} B=g-\operatorname{dim} H$. Furthermore, $B$ is isogenous to $E^{g-\operatorname{dim} H}$. Hence we may assume that $\psi$ is a surjective map $E^{g} \rightarrow E^{g-\operatorname{dim} H}$. Since $g-\operatorname{dim} H \geq r$ we may replace $\psi$ by the composition of $\psi$ with the projection onto the first $r$ factors of $E^{g-\operatorname{dim} H}$ to obtain a surjective morphism $E^{g} \rightarrow E^{r}$ vanishing at $p$.

Let $X$ be an irreducible closed subvariety of $E^{g}$ defined over $\overline{\mathbf{Q}}$. Let us assume $X^{\text {oa }} \neq \emptyset$. In view of Proposition 1 and the preceding paragraph, it suffices to show that (21) holds for $A=E^{g}$ and $B=E^{\operatorname{dim} X}$ in order to prove Proposition 2.

Deducing a height bound on $X^{\text {oa }} \cap\left(E^{g}\right)^{[\text {dim } X]}$ from one on $U(\overline{\mathbf{Q}}) \cap$ $\left(E^{g}\right)^{[\operatorname{dim} X]}$ involves handling the irreducible components of $\overline{X^{\text {oa }} \backslash U}$. These have dimension strictly less than $\operatorname{dim} X$ and in an unpublished manuscript the author shows that they can be treated using an inductive argument. We will not include this argument since proving a height bound for $X^{\text {oa }}$ can be done more naturally with the methods introduced in later sections.

An essential ingredient in the proof of Proposition 2, and our main result for general abelian varieties, is a special case of Ax's theorem. We will use it to show the existence of an algebraic obstruction subgroup in case hypothesis (21) is not satisfied.

Let $A$ be an abelian variety; we regard $A(\mathbf{C})$ as a complex Lie group and let $\exp _{A}: T(A) \rightarrow A(\mathbf{C})$ be the exponential map. A connected analytic subgroup of $A(\mathbf{C})$ is the image under $\exp _{A}$ of a complex vector subspace of $T(A)$. In general, a connected analytic subgroup need not be closed in the complex topology.

Theorem (Ax). Let $A$ be an abelian variety defined over $\mathbf{C}$ and let $G$ be a connected analytic subgroup of $A(\mathbf{C})$. Let $K$ be an irreducible analytic subvariety of an open subset (in the complex topology) of $A(\mathbf{C})$ with $K \subset G$ and $0 \in K$. Let $Z$ be the Zariski closure of $K$ in $A$ and let $H$ be the smallest algebraic subgroup of A containing $Z$, then

$$
\operatorname{dim} H \leq \operatorname{dim} Z+\operatorname{dim} G-\operatorname{dim} K .
$$

Proof. This is [1, Corollary 1]. Actually, Ax's result holds for all algebraic groups defined over $\mathbf{C}$. 
From now on we suppose that $E$ and $X$ are as in Proposition 2 and assume $r=\operatorname{dim} X \geq 1$. Since $E$ does not have complex multiplication we may identify $\operatorname{Hom}\left(E^{g}, E^{r}\right)$ with $\operatorname{Mat}_{r g}(\mathbf{Z}), \operatorname{Hom}\left(E^{g}, E^{r}\right) \otimes \mathbf{R}$ with $\operatorname{Mat}_{r g}(\mathbf{R})$, and $\operatorname{Hom}\left(E^{g}, E^{r}\right)_{\mathbf{R}}^{*}$ with matrices in $\operatorname{Mat}_{r g}(\mathbf{R})$ of maximal rank. Furthermore, we identify $T\left(E^{g}\right)$ with $\mathbf{C}^{g}$ and $T\left(E^{r}\right)$ with $\mathbf{C}^{r}$.

Let $\mathscr{L}_{g}, \mathscr{L}_{r}$ be symmetric and ample line bundles on $E^{g}, E^{r}$ respectively. For $\varphi \in \operatorname{Mat}_{r g}(\mathbf{Z})$ let $v_{X}(\varphi)=v_{X}^{r}(\varphi)$ be as in Sect. 5. Recall that $\varphi \mapsto v_{X}(\varphi)$ is a polynomial map on $\operatorname{Mat}_{r g}(\mathbf{R})$. For $\varphi \in \operatorname{Mat}_{r g}(\mathbf{Z})$, expression (16) simplifies to

$$
v_{X}(\varphi)=\operatorname{deg}\left(\left.\varphi\right|_{X}\right)\left(c_{1}\left(\mathcal{L}_{r}\right)^{r}\left[E^{r}\right]\right)
$$

indeed equality follows by Lemma 6(iii) if $\varphi(X)=E^{r}$, and both sides are 0 if $\varphi(X) \neq E^{r}$ because then $\operatorname{dim} \varphi(X)<\operatorname{dim} X$ and thus $\operatorname{deg}\left(\left.\varphi\right|_{X}\right)=0$. The $r$-fold self-intersection number $\left(c_{1}\left(\mathscr{L}_{r}\right)^{r}\left[E^{r}\right]\right)$ is a positive integer independent of $\varphi$, so $v_{X}(\varphi)$ is a positive integral multiple of $\operatorname{deg}\left(\left.\varphi\right|_{X}\right)$.

For the rest of this section any reference to a topology, for example on $E^{g}(\mathbf{C})$ or $\mathbf{C}^{g}$, will mean the complex topology unless stated otherwise; with the exception that irreducible will mean irreducible with respect to the Zariski topology.

Our first lemma is based on a well-known and easy to prove fact from the geometry of numbers: let $\Omega \subset \mathbf{R}^{n}$ be a discrete subgroup and $B \subset \mathbf{R}^{n}$ a bounded open neighborhood of 0 . There exist constants $c, C>0$ depending on $\Omega$ and $B$ such that the cardinality satisfies

$$
c \lambda^{\mathrm{rk} \Omega} \leq \#(\lambda B) \cap \Omega \leq C \lambda^{\mathrm{rk} \Omega} \text { for all } \lambda \geq 1 .
$$

Say $\exp _{g}: \mathbf{C}^{g} \rightarrow E^{g}(\mathbf{C})$ is the exponential map. It is open, surjective, holomorphic, and a homomorphism of groups. Furthermore, it is locally biholomorphic at 0 . Naturally, a corresponding map $\exp _{r}$ exists for $E^{r}(\mathbf{C})$. We note that an element $\varphi \in \operatorname{Mat}_{r g}(\mathbf{R})$ may not define a homomorphism $E^{g} \rightarrow E^{r}$ even after multiplication by a non-zero scalar. But it does define a linear map $\mathbf{C}^{g} \rightarrow \mathbf{C}^{r}$ which we also denote with $\varphi$.

If $M$ is a complex manifold and $z \in M$, we let $T_{z}(M)$ denote the holomorphic tangent space at $z$. If $\varphi$ is a holomorphic map between two complex manifolds we let $T_{z}(\varphi)$ denote the induced $\mathbf{C}$-linear map between corresponding tangent spaces. This construction is functorial.

The kernel of $\exp _{r}$, i.e. the period lattice of $E^{r}$, is a discrete subgroup of $\mathbf{C}^{r}$ with rank $2 r$, we denote it with $\Omega$.

We now assume that

$$
0 \text { is a non-singular point of } X \text {. }
$$

In this case a neighborhood (in the complex topology) of 0 in $X(\mathbf{C})$ is a complex submanifold of $E^{g}(\mathbf{C})$. Since $\exp _{g}$ is locally biholomorphic, we may find a complex submanifold $M \subset \mathbf{C}^{g}$ of dimension $r$ which maps biholomorphic to a neighborhood of $0 \in X(\mathbf{C})$. After translating by an element of $\Omega$ we may assume $0 \in M$. 
Lemma 8. Let $\varphi_{0} \in \operatorname{Mat}_{r g}(\mathbf{R})$ such that $T_{0}\left(\left.\varphi_{0}\right|_{M}\right): T_{0}(M) \rightarrow T_{0}\left(\mathbf{C}^{r}\right)=\mathbf{C}^{r}$ is an isomorphism of $\mathbf{C}$-vector spaces. There exist a constant $C_{20}>0$ and an open neighborhood $W \subset \operatorname{Mat}_{r g}(\mathbf{R})$ of $\varphi_{0}$ such that if $\varphi \in W$ and $\lambda \geq 1$, then

$$
\#(\gamma+\lambda \varphi(M)) \cap \Omega \geq C_{20} \lambda^{2 r}
$$

for all $\gamma$ in some open neighborhood of $0 \in \mathbf{C}^{r}$ (which depends on $M$ and $\varphi_{0}$ but is independent of $\varphi$ and $\lambda$ ).

Proof. We consider the holomorphic map

$$
\Psi: \operatorname{Mat}_{r g}(\mathbf{C}) \times M \rightarrow \operatorname{Mat}_{r g}(\mathbf{C}) \times \mathbf{C}^{r} \quad \text { defined by } \Psi(\varphi, z)=(\varphi, \varphi(z)) .
$$

The induced map at $\left(\varphi_{0}, 0\right)$ on tangent spaces is an isomorphism because $T_{0}\left(\left.\varphi_{0}\right|_{M}\right)$ is one itself. Hence $\Psi$ is locally biholomorphic at 0 . In particular, there exist an open set $W^{\prime} \subset \operatorname{Mat}_{r g}(\mathbf{C})$ containing $\varphi_{0}$ and an open ball $B \subset \mathbf{C}^{r}$ of radius $\delta>0$ (with respect to the Hermitian norm $|\cdot|$ ) around 0 such that $W^{\prime} \times B \subset \Psi\left(\operatorname{Mat}_{r g}(\mathbf{C}) \times M\right)$. Hence

$$
B \subset \varphi(M) \text { for all } \varphi \in W=W^{\prime} \cap \operatorname{Mat}_{r g}(\mathbf{R}) .
$$

It follows from the lower bound in (31) that there exists a constant $C_{20}>0$ such that

$$
\#\left(\frac{\lambda}{2} B\right) \cap \Omega \geq C_{20} \lambda^{2 r} \quad \text { for all } \lambda \geq 1 ;
$$

here $C_{20}$ is independent of $\lambda$.

Let $\varphi \in W$ and $\lambda \geq 1$ and assume $\gamma \in B / 2$. There are at least $C_{20} \lambda^{2 r}$ distinct $\omega_{i} \in(\lambda B / 2) \cap \Omega$ and these satisfy $\left|\omega_{i}-\gamma\right|<\delta \lambda / 2+\delta / 2 \leq \delta \lambda$, thus $\omega_{i}-\gamma \in \lambda B$. From the inclusion (34) we conclude $\omega_{i} \in \gamma+\lambda \varphi(M)$ and the lemma follows with $B / 2$ the neighborhood alluded to in the assertion.

For a possibly reducible variety $Z$ containing a point $p$, we let $\operatorname{dim}_{p} Z$ denote the maximal dimension of an irreducible component of $Z$ passing through $p$.

The next remark is possibly well-known, but we provide a proof for lack of reference: Let $Z$ be an irreducible variety defined over $\mathbf{C}$ and $U \subset Z(\mathbf{C})$ an open and non-empty subset, then

the Zariski closure $\bar{U}$ equals $Z$.

Indeed, for $p \in U$ the dimension of $\bar{U}$ at $p$ equals the dimension of $Z$ at $p$, where we consider both as complex analytic spaces. This dimension is $\operatorname{dim}_{p} \bar{U}=\operatorname{dim}_{p} Z$ by GAGA. But $\bar{U} \subset Z$ and so we must have equality because $Z$ is irreducible.

The next lemma, whose proof involves Lemma 8, gives an important criterion for the non-vanishing of $v_{X}\left(\varphi_{0}\right)$. 
Lemma 9. Let $\varphi_{0}$ be as in Lemma 8, then $v_{X}\left(\varphi_{0}\right)>0$.

Proof. Say $C_{20}$ and $W$ are as given by Lemma 8 .

Let $\varphi \in W \cap \operatorname{Mat}_{r g}(\mathbf{Q})$. We choose a positive integer $\lambda$ with $\lambda \varphi \in$ Mat $_{r g}(\mathbf{Z})$. Let $\gamma$ be any point in the neighborhood of 0 supplied by Lemma 8 . By (33) there are $\Delta \geq C_{20} \lambda^{2 r}$ distinct periods $\omega_{1}, \ldots, \omega_{\Delta} \in \gamma+\lambda \varphi(M)$. In other words there exist $x_{i} \in M$ such that

$$
\omega_{i}=\gamma+\lambda \varphi\left(x_{i}\right) \quad \text { for } 1 \leq i \leq \Delta .
$$

The $x_{i}$ are distinct since the $\omega_{i}$ are themselves.

Now $\lambda \varphi$ is a homomorphism of algebraic groups $E^{g} \rightarrow E^{r}$ so the diagram

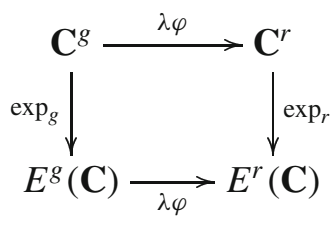

commutes. We apply $\exp _{r}$ to (36) and use the fact that the $\omega_{i}$ are periods to obtain $0=\exp _{r}\left(\omega_{i}\right)=\exp _{r}\left(\gamma+\lambda \varphi\left(x_{i}\right)\right)=\exp _{r}(\gamma)+\exp _{r}\left(\lambda \varphi\left(x_{i}\right)\right)=$ $\exp _{r}(\gamma)+(\lambda \varphi)\left(\exp _{g}\left(x_{i}\right)\right)$. Hence

$$
(\lambda \varphi)\left(\exp _{g}\left(x_{i}\right)\right)=-\exp _{r}(\gamma) .
$$

By construction, $\exp _{g}$ maps $M$ biholomorphically to a neighborhood of 0 in $X(\mathbf{C})$. Hence $\left.\exp _{g}\right|_{M}$ is injective and so $\exp _{g}\left(x_{1}\right), \ldots, \exp _{g}\left(x_{\Delta}\right)$ are distinct. Since these points lie in $X(\mathbf{C})$ we conclude from (37) that

$$
\left.\#(\lambda \varphi)\right|_{X} ^{-1}\left(-\exp _{r}(\gamma)\right) \geq \Delta \geq C_{20} \lambda^{2 r} .
$$

Recall that $\exp _{r}$ is an open map. Since $\gamma$ was arbitrary in a non-empty open subset of $\mathbf{C}^{r}$, the possible $-\exp _{r}(\gamma)$ form an open and hence Zariski dense subset of $E^{r}(\mathbf{C})$ by (35). There is $U \subset E^{r}$ Zariski open and nonempty such that $\operatorname{deg}\left(\left.(\lambda \varphi)\right|_{X}\right)=\left.\#(\lambda \varphi)\right|_{X} ^{-1}(u)$ for $u \in U(\mathbf{C})$. Hence at least one such $u$ is of the form $-\exp _{r}(\gamma)$. So (38) implies $\operatorname{deg}\left(\left.(\lambda \varphi)\right|_{X}\right) \geq C_{20} \lambda^{2 r}$. We saw in (30) that $v_{X}(\lambda \varphi)$ equals $\operatorname{deg}\left(\left.(\lambda \varphi)\right|_{X}\right)$ times a positive integer. Therefore $v_{X}(\lambda \varphi) \geq \operatorname{deg}\left(\left.(\lambda \varphi)\right|_{X}\right) \geq C_{20} \lambda^{2 r}$. Since $v_{X}(\lambda \varphi)=\lambda^{2 r} v_{X}(\varphi)$ by homogeneity (15) we conclude

$$
v_{X}(\varphi) \geq C_{20}>0
$$

after cancelling $\lambda$.

Inequality (39) holds for all $\varphi \in W \cap \operatorname{Mat}_{r g}(\mathbf{Q})$. Since this set lies dense in $W$ and because $v_{X}$ is continuous we have $v_{X}\left(\varphi_{0}\right) \geq C_{20}$. In particular, $v_{X}\left(\varphi_{0}\right)>0$.

We need a result on holomorphic maps between complex manifolds. 
Lemma 10. Let $M \subset \mathbf{C}^{g}$ be any complex submanifold, let $f: M \rightarrow \mathbf{C}^{r}$ be a holomorphic map, and say $z_{0} \in M$. Assume that the rank of $T_{z}(f)$ : $T_{z}(M) \rightarrow \mathbf{C}^{r}$ is at most $\operatorname{dim} M-1$ for all $z$ in an open neighborhood of $z_{0}$. Then there exists a non-empty open subset $U \subset M$ such that $f^{-1}(f(z)) \cap U$ is a complex submanifold of $\mathbf{C}^{g}$ of positive dimension for all $z \in U$.

Proof. We can find a non-empty open neighborhood $U \subset M$ of $z_{0}$ such that $T_{z}(f)$ has constant rank at most $\operatorname{dim} M-1$ for all $z \in U$. The lemma follows from [25, Corollary 7F, p. 314].

The next lemma applies Lemma 9 and Ax's theorem to derive an algebraic consequence if the analytic hypothesis (21) is not satisfied.

Lemma 11. Assume that there exists $\varphi_{0} \in \operatorname{Mat}_{r g}(\mathbf{R})$ with maximal rank such that $v_{X}\left(\varphi_{0}\right)=0$. Then there is an open non-empty subset $V \subset X(\mathbf{C})$ such that for any $p \in V$ there exists an irreducible algebraic subgroup $H \subset E^{g}$ with

$$
\operatorname{dim}_{p} X \cap(p+H) \geq \max \{1, r+\operatorname{dim} H-g+1\} .
$$

Moreover, we can take $H$ to have dimension $g-r$.

Proof. By Lemma 9 the linear map $T_{z}\left(\left.\varphi_{0}\right|_{M}\right)$ has rank at most $r-1$ for $z=0$. This inequality for the rank must hold for all $z$ in a neighborhood of 0 in order to apply Lemma 10. We show this by applying Lemma 6(ii) which tells us that $v_{X-p}\left(\varphi_{0}\right)=0$ for all $p \in E^{g}(\mathbf{C})$. After replacing $M$ by $M-z$ with any $z \in M$ we conclude from Lemma 9 that $T_{0}\left(\left.\varphi_{0}\right|_{M-z}\right)$ has rank at most $r-1$. Equivalently, $T_{z}\left(\left.\varphi_{0}\right|_{M}\right)$ has rank at most $r-1$ for all $z \in M$.

By Lemma 10 there is an open non-empty $U \subset M$ such that the intersection of $U-z$ with $\operatorname{ker} \varphi_{0}$, the kernel of $\varphi_{0}$, is a complex submanifold of $\mathbf{C}^{g}$ of positive dimension for all $z \in U$. After shrinking $U$ we may assume that $\exp _{g}$ is biholomorphic when restricted to $U-z$ for all $z \in U$.

Now $G=\exp _{g}\left(\operatorname{ker} \varphi_{0}\right)$ is a connected analytic subgroup of $E^{g}(\mathbf{C})$. Since $\varphi_{0}$ has rank $r$, the dimension of $G$ is $g-r$. Let $z \in U$ and $p=\exp _{g}(z)$. Then $\exp _{g}\left((U-z) \cap \operatorname{ker} \varphi_{0}\right)$ contains $K$, a connected complex submanifold of $E^{g}(\mathbf{C})$ of positive dimension with $0 \in K$. Let $Z$ be the Zariski closure of $K$ in $E^{g}$, it is contained in $X-p$ and by [12, theorem on p. 168] it is irreducible. Finally, let $H$ be the minimal algebraic subgroup containing $Z$. Ax's theorem implies $\operatorname{dim} H \leq \operatorname{dim} Z+\operatorname{dim} G-\operatorname{dim} K \leq \operatorname{dim} Z+g-r-1$. We have $p \in p+Z$ and $p+Z \subset p+H$, furthermore from $\operatorname{dim} Z \geq \operatorname{dim} K \geq 1$ we conclude

$$
\operatorname{dim} Z \geq \max \{1, r+\operatorname{dim} H-g+1\} .
$$

The first claim of the lemma follows with $V=\exp _{g}(U)$ using the fact that $\exp _{g}$ is an open map.

We now show that $H$ can be chosen with $\operatorname{dim} H=g-r$ such that (40) still holds after possibly shrinking $Z$. 
If $\operatorname{dim} H>g-r$, then we can find an algebraic subgroup $H^{\prime} \subset E^{g}$ with $\operatorname{dim} H \cap H^{\prime}=g-r$ and $\operatorname{dim} H^{\prime}=2 g-r-\operatorname{dim} H$ : indeed $H$ is an irreducible component of a fibre of some homomorphism $E^{g} \rightarrow E^{g-\operatorname{dim} H}$ which is represented by an element of $\operatorname{Mat}_{g-\operatorname{dim} H, g}(\mathbf{Z})$. The kernel of any matrix in $\mathbf{M a t}_{r+\operatorname{dim} H-g, g}(\mathbf{Z})$ in general position determines the required algebraic subgroup $H^{\prime}$. The intersection $Z \cap H^{\prime}$ is non-empty since it contains 0 . Thus it contains an irreducible component $Z^{\prime}$ which satisfies $\operatorname{dim} Z^{\prime} \geq$ $\operatorname{dim} Z+\operatorname{dim} H^{\prime}-g \geq r+\operatorname{dim} H+\operatorname{dim} H^{\prime}-2 g+1=1$, cf. [11, p. 137]. The lemma follows in this case from $Z^{\prime} \subset H \cap H^{\prime}$.

If $\operatorname{dim} H \leq g-r$, we choose an algebraic subgroup $H^{\prime} \supset H$ with $\operatorname{dim} H^{\prime}=g-r$. Then $\operatorname{dim} Z \geq 1=\max \left\{1, r+\operatorname{dim} H^{\prime}-g+1\right\}$.

We note that the assertion of the last lemma implies that $X(\mathbf{C}) \backslash X^{\text {oa }}$ contains $V$. Now $V$, being non-empty and open, is Zariski dense in $X$ by (35).

Proof of Proposition 2. Let $E$ and $X$ be as in the hypothesis and let us suppose $r=\operatorname{dim} X \geq 1$. As stated near the beginning of this section, it suffices to verify (21) for $A=E^{g}$ and $B=E^{r}$ in order to prove the proposition.

Let us fix a non-singular $p \in X(\mathbf{C})$, then (32) holds with $X$ replaced by $X^{\prime}=X-p$. A straight-forward argument using the definition of $X^{\text {oa }}$ shows that $X^{\prime \text { oa }}=X^{\text {oa }}-p$. So by hypothesis we have $X^{\prime \text { oa }} \neq \emptyset$. By Rémond's result $X^{\prime \text { oa }}$ is Zariski open in $X^{\prime}$. Therefore, $X^{\prime} \backslash X^{\prime \text { oa }}$ is not Zariski dense in $X^{\prime}$. By Lemma 11 and the comment directly after its proof, $v_{X^{\prime}}(\varphi)>0$ for any $\varphi \in$ Mat $_{r g}(\mathbf{R})$ of maximal rank. But $v_{X^{\prime}}=v_{X}$ by Lemma 6(ii) therefore $X$ satisfies (21) with $A=E^{g}$ and $B=E^{r}$.

Some comments are in order. Let $X \subset E^{g}$ be any irreducible closed subvariety of dimension $r \geq 1$ defined over $\mathbf{C}$ such that $v_{X}\left(\varphi_{0}\right)=0$ for some $\varphi_{0} \in \mathrm{Mat}_{r g}(\mathbf{R})$ of maximal rank. As in the proof of Proposition 2 we may translate $X$ by a point of $E^{g}(\mathbf{C})$ without changing the value of $v_{X}\left(\varphi_{0}\right)$. Thus we assume $0 \in X(\mathbf{C})$ is non-singular. We conclude from Lemma 11 that the union over all

$$
Z_{H}=\left\{p \in X(\mathbf{C}) ; \operatorname{dim}_{p} X \cap(p+H) \geq 1\right\},
$$

where $H$ runs through all algebraic subgroups of $E^{g}$ with dimension $g-r$, contains a non-empty open subset of $X(\mathbf{C})$. Further down (cf. Lemmas 22 and 23) we will use the fact that each $Z_{H}$ is Zariski closed in $X$ and that $E^{g}$ has only countably many algebraic subgroups to see that $X=Z_{H}$ for a single $H$. This immediately implies $X^{\text {oa }}=\emptyset$. Such an argument can be used in the last paragraph of the proof of Proposition 2 to avoid using Rémond's result on the Zariski-openness of $X^{\text {oa }}$.

Say $\varphi: E^{g} \rightarrow E^{r}$ is a surjective homomorphism whose kernel contains an algebraic subgroup $H$ with finite index. The equality $X=Z_{H}$ entails that the fibres of $\left.\varphi\right|_{X}$ have dimension at least 1 . The theorem on the dimension of the fibres implies that $\varphi(X)$ is not Zariski dense in $E^{r}$. Hence $\operatorname{deg}\left(\left.\varphi\right|_{X}\right)=0$ 
and so $v_{X}(\varphi)=0$. As $\varphi$ is represented by an element of $\operatorname{Mat}_{r g}(\mathbf{Z})$ of maximal rank we obtain the non-trivial direction in the following Hasse principle:

Lemma 12. The polynomial $v_{X}$ vanishes at an element of $\operatorname{Mat}_{r g}(\mathbf{R})$ of maximal rank if and only if it vanishes at an element of $\mathbf{M a t}_{r g}(\mathbf{Q})$ of maximal rank.

As we have seen above (30), the map $v_{X}$ takes on a simplified form since $\operatorname{dim} X=\operatorname{dim} E^{r}$. We claim that it is invariant under the natural leftaction of $\mathrm{SL}_{r}(\mathbf{C})$ on $\operatorname{Mat}_{r g}(\mathbf{C})$. Indeed, if $\gamma \in \mathrm{SL}_{r}(\mathbf{Z})$ then $\gamma$ induces an automorphism of $E^{r}$. It is not hard to $\operatorname{see} \operatorname{deg}\left(\left.\gamma \varphi\right|_{X}\right)=\operatorname{deg}\left(\left.\varphi\right|_{X}\right)$ for all $\varphi \in \operatorname{Mat}_{r g}(\mathbf{Z})$. Because $v_{X}$ equals the degree up-to a non-zero factor we get $v_{X}(\gamma \varphi)=v_{X}(\varphi)$. We recall that $v_{X}$ is polynomial; since $\operatorname{Mat}_{r g}(\mathbf{Z})$ is Zariski dense in $\operatorname{Mat}_{r g}(\mathbf{C})$ and $\mathrm{SL}_{r}(\mathbf{Z})$ is Zariski dense in $\mathrm{SL}_{r}(\mathbf{C})$ we get $v_{X}(\gamma \varphi)=v_{X}(\varphi)$ for all $\varphi \in \operatorname{Mat}_{r g}(\mathbf{C})$ and $\gamma \in \operatorname{SL}_{r}(\mathbf{C})$, proving our claim.

By a result of Weyl from invariant theory [24, Theorem 2.6.A, p. 45], we can express $v_{X}(\varphi)$ as $\beta_{X}\left(\Delta_{0}, \ldots, \Delta_{N}\right)$, the value of a polynomial $\beta_{X}$ in rational coefficients where $\Delta_{i}$ are the determinants of the maximal minors of $\varphi$; here $N+1=\left(\begin{array}{l}g \\ r\end{array}\right)$ is the number of such minors. Since $v_{X}$ is homogeneous of degree $2 r$ we can take $\beta_{X}$ to be homogeneous of degree 2 . We will restate Lemma 12 in terms of $\beta_{X}$. The orbits of the left-action of $\mathrm{GL}_{r}(\mathbf{C})$ on elements of $\mathrm{Mat}_{r g}(\mathbf{C})$ of maximal rank can be identified with complex points of the Grassmannian variety $G(r, g)$ which parametrize $r$-dimensional vector subspaces of $\mathbf{C}^{g}$. The $\Delta_{i}$ are the projective coordinates of the Plücker embedding $G(r, g) \hookrightarrow \mathbf{P}^{N}$; we will therefore assume $G(r, g) \subset \mathbf{P}^{N}$.

We note that if $1<r<g-1$, then the $\Delta_{i}$ satisfy Plücker relations which are homogeneous polynomials of degree 2 . So $\beta_{X}$ is not uniquely determined by $X$. If $r=1$ or $r=g-1$ then the $\Delta_{i}$ do not satisfy any Plücker relations, hence $G(r, g)=\mathbf{P}^{N}$, and so $\beta_{X}$ is uniquely determined.

Of course, the value of $\beta_{X}$ at a point of $G(r, g)(\mathbf{C})$ is not well-defined. But the sign of $\beta_{X}$, which can be either 0 or \pm 1 , at a point of $G(r, g)(\mathbf{R})$ is because $\beta_{X}$ is homogeneous of degree 2 and has real coefficients. Lemma 12 can be reformulated as follows.

Lemma 12'. The quadratic form $\beta_{X}$ vanishes at an element of $G(r, g)(\mathbf{R})$ if and only if it vanishes at an element of $G(r, g)(\mathbf{Q})$.

If $r=1$ or $r=g-1$ then as was stated above we have $G(r, g)=\mathbf{P}^{g-1}$. The case $r=1$ corresponds to curves in $E^{g}$ and hence Viada's theorem [23]. An elementary argument from the theory of quadratic forms shows that if $\beta$ is any quadratic form in rational coefficients and $g$ variables then

(41) $\beta$ is positive on $\mathbf{R}^{g} \backslash\{0\}$ if and only if it is positive on $\mathbf{Q}^{g} \backslash\{0\}$.

So if $X$ is a curve or a hypersurface, then Lemma 12 (and 12') follows without invoking Ax's theorem. Moreover, the same can be said about Proposition 2. 
Now say $1<r<g-1$ and let $\beta$ be a quadratic form in $N+1$ variables and rational coefficients, statement (41) raises the following question: if $\beta$ is positive on $G(r, g)(\mathbf{Q}) \subset \mathbf{P}^{N}(\mathbf{Q})$ does it follow that $\beta$ is positive on $G(r, g)(\mathbf{R})$ ?

The answer is already no in the simplest case, that is if $r=2$ and $g=4$ (this corresponds to a surface in $E^{4}$ ); here $G(2,4)$ is a hypersurface of degree 2 . After suitably ordering the $\Delta_{i}$ they satisfy the Plücker relation

$$
\Delta_{0} \Delta_{5}-\Delta_{1} \Delta_{4}+\Delta_{2} \Delta_{3}=0
$$

The quadratic form

$$
\beta=\left(\Delta_{0}-2 \Delta_{5}\right)^{2}+\left(\Delta_{1}-\Delta_{4}\right)^{2}+\Delta_{2}^{2}+\Delta_{3}^{2}
$$

has rational coefficients and is certainly never negative on $G(2,4)(\mathbf{R})$. Moreover, because $\sqrt{2}$ is irrational it does not vanish on $G(2,4)(\mathbf{Q})$ and is therefore positive on this set. On the other hand, $\beta$ vanishes at $[2: \sqrt{2}$ : $0: 0: \sqrt{2}: 1] \in G(2,4)(\mathbf{R})$. In particular, it is not of the form $\beta_{X}$ for any surface $X$ in $E^{4}$.

Thus for arbitrary $\beta$, statement (41) cannot generalize to Grassmannians. And it seems that Ax's theorem is indispensable in the proof of Proposition 2 (and the bounded height theorem).

\section{Counting lattice points}

The main result of this section, Proposition 3, is a more flexible version of Lemma 8. It is a lattice point counting result for possibly non-convex sets which expand in a certain special way.

In this section any reference to a topology on a real or complex vector space will always mean the Hausdorff topology coming from a norm.

Proposition 3. Let $X$ and $Y$ be finite dimensional $\mathbf{C}$-vector spaces, let $M \subset X$ and $N \subset Y$ be complex submanifolds containing 0 with $\operatorname{dim} M+$ $\operatorname{dim} N=\operatorname{dim} Y$, and let $\Omega \subset Y$ be a discrete subgroup. Say $\varphi_{0} \in$ $\operatorname{Hom}(X, Y)$ and assume that $T_{0}(\phi): T_{0}(M \times N) \rightarrow T_{0}(Y)$ is an isomorphism where $\phi: M \times N \rightarrow Y$ is the holomorphic map defined by $\phi(x, y)=\varphi_{0}(x)-y$. There exist a constant $C_{21}>0$ and an open neighborhood $W \subset \operatorname{Hom}(X, Y)$ of $\varphi_{0}$ such that if $\varphi \in W$ and $\lambda \geq 1$, then

$$
\#(\gamma+\lambda \varphi(M)-N) \cap \Omega \geq C_{21} \lambda^{2 \operatorname{dim} M+\mathrm{rk} \Omega-2 \operatorname{dim} Y}
$$

for all $\gamma$ in some non-empty open subset of $Y$ (which may depend on $\varphi$ and $\lambda$ ).

In our application $Y$ and $\Omega$ will be the tangent space, respectively period lattice of an abelian variety; hence $\Omega$ will have maximal possible rank $2 \operatorname{dim} Y$. Keeping in mind applications to the period lattice of a semi-abelian 
variety, we also provide a counting result for discrete subgroups of nonmaximal rank.

Apart from the property of the open set containing $\gamma$, Lemma 8 follows from the proposition on taking $N=\{0\}$.

Since $\phi(0)=0$ it is natural to ask if one can assume that the open subset of $Y$ alluded to in the proposition contains 0 . An argument leading to a negative answer was suggested by the referee who has kindly agreed to its appearance here.

In the situation of the proposition, let $X=Y=\mathbf{C}^{n}$ and say $\varphi_{0}$ is the identity. We take as $M, N \subset \mathbf{C}^{n}$ the complex submanifolds $\left\{\left(z_{1}, \ldots, z_{n-1}, z_{1}^{2}+\right.\right.$ $\left.\cdots+z_{n-1}^{2}\right) ; z_{i} \in \mathbf{C}$ with $\left.\left|z_{i}\right|<1\right\}$ and $\left\{\left(0, \ldots, 0, z_{n}\right) ; z_{n} \in \mathbf{C}\right.$ with $\left.\left|z_{n}\right|<1\right\}$ respectively. Then $\operatorname{dim} M=n-1, \operatorname{dim} N=1$, and $T_{0}(M \times N) \rightarrow \mathbf{C}^{n}$ as defined in the proposition is an isomorphism. Finally, we choose $\Omega=$ $\mathbf{Z}^{n-1} \times\{0\} \subset \mathbf{C}^{n}$. Say now $\lambda \geq 1$ and $\left(\omega_{1}, \ldots, \omega_{n-1}, 0\right) \in(\lambda M-N) \cap \Omega$; this corresponds to the choice $\gamma=0$ and $\varphi$ the identity in (42). Then $\omega_{i}=\lambda z_{i}$ for $1 \leq i \leq n-1$ and $\lambda^{-1}\left(\omega_{1}^{2}+\cdots+\omega_{n-1}^{2}\right)=-z_{n}$ with $\left|z_{n}\right|<1$. We get $\left|\omega_{i}\right|<\lambda^{1 / 2}$ leaving at most $\left(2 \lambda^{1 / 2}+1\right)^{n-1}$ possibilities for the integral vector $\left(\omega_{1}, \ldots, \omega_{n-1}, 0\right)$. But rk $\Omega-2=n-3$, the exponent on the right-hand side of (42), exceeds $(n-1) / 2$ if $n \geq 6$. So (42) is impossible for $\gamma=0$ and large $\lambda$.

It would be interesting to know if such an example is possible for $\Omega$ of maximal rank $2 \operatorname{dim} Y$.

In view of its application, the proposition is formulated for complex manifolds, but its proof is based on Lemma 13 which is a statement on smooth real functions. We begin by introducing some notation and conventions applying to this section.

Let $|\cdot|$ denote the euclidean norm on $\mathbf{R}^{n}$ and also the operator norm on the vector space of matrices with real coefficients with respect to the euclidean norm. Let $B_{r}(x) \subset \mathbf{R}^{n}$ be the open ball of radius $r$ around $x \in \mathbf{R}^{n}$. For a differentiable map $\phi: U \rightarrow \mathbf{R}^{m}$ on an open subset $U \subset \mathbf{R}^{n}$ we define $J_{x}(\phi) \in \operatorname{Mat}_{m n}(\mathbf{R})$ to be the Jacobian matrix at $x \in U$. We call $\phi$ a smooth map if it is infinitely often differentiable.

For $i=1,2$ let $n_{i}$ and $m_{i}$ be non-negative integers and $\phi_{i}: U_{i} \rightarrow \mathbf{R}^{m_{i}}$ a smooth map on an open set $U_{i} \subset \mathbf{R}^{n_{i}}$ containing 0 such that $\phi_{i}(0)=0$. We assume $m_{2} \geq n_{1}$ and let $n_{2}=m_{2}-n_{1}$; if $n_{2}=0$ then $\mathbf{R}^{n_{2}}=\{0\}$. For any $\varphi \in \operatorname{Mat}_{m_{2} m_{1}}(\mathbf{R})$ we define a smooth map $\phi_{\varphi}: U_{1} \times U_{2} \rightarrow \mathbf{R}^{m_{2}}$ by setting

$$
\phi_{\varphi}\left(u_{1}, u_{2}\right)=\varphi\left(\phi_{1}\left(u_{1}\right)\right)-\phi_{2}\left(u_{2}\right) .
$$

Finally, let $\Omega$ be a discrete subgroup of $\mathbf{R}^{m_{2}}$.

Lemma 13. Let $\varphi_{0} \in \operatorname{Mat}_{m_{2} m_{1}}(\mathbf{R})$ such that $J_{0}\left(\phi_{\varphi_{0}}\right)$ is invertible. There exist $C_{27}>0$ and an open neighborhood $W \subset \operatorname{Mat}_{m_{2} m_{1}}(\mathbf{R})$ of $\varphi_{0}$ such that if $\varphi \in W$ and $\lambda \geq 1$, then

$$
\#\left(\gamma+\phi_{\lambda \varphi}\left(U_{1} \times U_{2}\right)\right) \cap \Omega \geq C_{27} \lambda^{n_{1}+\mathrm{rk} \Omega-m_{2}}
$$


for all $\gamma$ in some non-empty open subset of $\mathbf{R}^{m_{2}}$ (which may depend on $\varphi$ and $\lambda$ ).

We note that the lemma follows directly if $n_{1}=0$ since then $n_{1}+$ rk $\Omega-$ $m_{2}=\operatorname{rk} \Omega-m_{2} \leq 0$. We assume $n_{1} \geq 1$, hence $m_{2}=n_{1}+n_{2} \geq 1$. Now the hypothesis that $J_{0}\left(\phi_{\varphi_{0}}\right)$ is invertible forces $m_{1} \geq 1$. So we will assume that $m_{1}, m_{2}$, and $n_{1}$ are positive, but we shall not assume this for $n_{2}$.

The proof of Lemma 13 is done in two steps. It turns out to be easier to count points of $\Omega$ contained in a "tubular neighborhood" (cf. (43)) of $\lambda \varphi\left(\phi_{1}\left(U_{1}\right)\right)$ with fixed radius than in $\phi_{\lambda \varphi}\left(U_{1} \times U_{2}\right)$. In the first step, Lemma 15, we bound the number of points in $\Omega$ contained in such a neighborhood from below in terms of $\lambda$. Then in the second step we show that for $\varphi$ near $\varphi_{0}$ and $\lambda \geq 1$, the set of points $\phi_{\lambda \varphi}\left(U_{1} \times U_{2}\right)$ contains a tubular neighborhood of $\lambda \phi_{1}\left(U_{1}\right)$ of radius not depending on $\varphi$ or $\lambda$. This is done in Lemma 17 whose proof uses Lemma 14 which is an effective variant of the inverse function theorem:

Lemma 14. Let $U \subset \mathbf{R}^{m}$ be open and let $\phi: U \rightarrow \mathbf{R}^{m}$ be smooth. Let $x \in U$ such that $J_{x}(\phi)$ is invertible. Let $r$ be a positive real with $\overline{B_{r}(x)} \subset U$ and such that $x^{\prime}, x^{\prime \prime} \in \overline{B_{r}(x)}$ implies

$$
\left|J_{x}(\phi)^{-1}\left(J_{x^{\prime}}(\phi)-J_{x^{\prime \prime}}(\phi)\right)\right| \leq 1 / 2 .
$$

If $\beta=1 /\left|J_{x}(\phi)^{-1}\right|$, then

$$
\overline{B_{\beta r / 2}(\phi(x))} \subset \phi\left(\overline{B_{r}(x)}\right) .
$$

Proof. This is [14, Lemma 5.4, p. 17] with $f(z)=J_{x}(\phi)^{-1}(\phi(z+x)-\phi(x))$.

For any subset $S \subset \mathbf{R}^{n}$ and for any $\epsilon>0$ we define the tubular neighborhood of radius $\epsilon$ around $S$ as

$$
\mathcal{N}_{\epsilon}(S)=\left\{x \in \mathbf{R}^{n} ; \text { there exists } y \in S \text { with }|x-y|<\epsilon\right\} .
$$

A first consequence is a uniform lower bound for the number of points in $\Omega$ contained in a fixed tubular neighborhood as $\varphi$ and $\lambda$ vary.

Lemma 15. Assume $\varphi_{0} \in \operatorname{Mat}_{m_{2} m_{1}}(\mathbf{R})$ such that $J_{0}\left(\varphi_{0} \circ \phi_{1}\right)$ has rank $n_{1}$. There is an open set $W \subset \operatorname{Mat}_{m_{2} m_{1}}(\mathbf{R})$ containing $\varphi_{0}$ with the following property: for any $\epsilon>0$ there exists $C_{25}>0$ such that if $\varphi \in W$ and $\lambda \geq 1$, then

$$
\#\left(\gamma+\mathcal{N}_{\epsilon}\left(\lambda \varphi\left(\phi_{1}\left(U_{1}\right)\right)\right)\right) \cap \Omega \geq C_{25} \lambda^{n_{1}+\mathrm{rk} \Omega-m_{2}}
$$

for all $\gamma$ in some non-empty open subset of $\mathbf{R}^{m_{2}}$ (which may depend on $\varphi$ and $\lambda$ ). 
Proof. We note that it suffices to prove the lower bound for a single $\gamma$ : if (44) holds for $\gamma_{0}$ and with $\epsilon$ replaced by $\epsilon / 2$, then (44) holds for all $\gamma$ with $\left|\gamma-\gamma_{0}\right|<\epsilon / 2$. After shrinking $U_{1}$ we may assume $\left|\varphi_{0}\left(\phi_{1}(u)\right)\right| \leq 1 / 2$ for all $u \in U_{1}$.

By hypothesis the image $\operatorname{im}\left(J_{0}\left(\varphi_{0} \circ \phi_{1}\right)\right) \subset \mathbf{R}^{m_{2}}$ of the linear map $J_{0}\left(\varphi_{0} \circ \phi_{1}\right)$ has dimension $n_{1} \leq m_{2}$. We choose an auxiliary vector space $V \subset \mathbf{R}^{m_{2}}$ of dimension $m_{2}-n_{1}$ with

$$
V \cap \operatorname{im}\left(J_{0}\left(\varphi_{0} \circ \phi_{1}\right)\right)=0 .
$$

Just for the rest of this proof let $\phi_{2}: \mathbf{R}^{m_{2}-n_{1}} \rightarrow V$ be an isomorphism of real vector spaces (and $U_{2}=\mathbf{R}^{m_{2}-n_{1}}$ ).

By (45) the matrix $J_{0}\left(\phi_{\varphi_{0}}\right)$ is invertible. There is an open neighborhood $W \subset \operatorname{Mat}_{m_{2} m_{1}}(\mathbf{R})$ of $\varphi_{0}$ and a constant $C_{22}>0$ such that $J_{0}\left(\phi_{\varphi}\right)$ is invertible and $\left|J_{0}\left(\phi_{\varphi}\right)^{-1}\right| \leq C_{22}$ for all $\varphi \in W$. By smoothness of $\phi_{1}$ and $\phi_{2}$ and on possibly shrinking $W$ we can find $r>0$ such that $\left|J_{x^{\prime}}\left(\phi_{\varphi}\right)-J_{x^{\prime \prime}}\left(\phi_{\varphi}\right)\right| \leq$ $1 /\left(2 C_{22}\right)$ for all $x^{\prime}, x^{\prime \prime} \in \overline{B_{r}(0)}$ and all $\varphi \in W$. Hence

$$
\left|J_{0}\left(\phi_{\varphi}\right)^{-1}\left(J_{x^{\prime}}\left(\phi_{\varphi}\right)-J_{x^{\prime \prime}}\left(\phi_{\varphi}\right)\right)\right| \leq\left|J_{0}\left(\phi_{\varphi}\right)^{-1}\right|\left|J_{x^{\prime}}\left(\phi_{\varphi}\right)-J_{x^{\prime \prime}}\left(\phi_{\varphi}\right)\right| \leq 1 / 2
$$

for all $x^{\prime}, x^{\prime \prime} \in \overline{B_{r}(0)}$ and all $\varphi \in W$. We may assume $\overline{B_{r}(0)} \subset U_{1} \times \mathbf{R}^{m_{2}-m_{1}}$. After shrinking $W$ again we may assume

$$
\left|\varphi\left(\phi_{1}(u)\right)\right| \leq 1 \quad \text { for all } \varphi \in W \text { and all } u \in U_{1} .
$$

By Lemma 14 there exists $\epsilon_{0}>0$ with

$$
B_{\epsilon_{0}}(0) \subset \phi_{\varphi}\left(\overline{B_{r}(0)}\right) \subset \phi_{\varphi}\left(U_{1} \times \mathbf{R}^{m_{2}-n_{1}}\right) \quad \text { for all } \varphi \in W .
$$

We may suppose $\epsilon_{0} \leq 1$.

In order to prove the lemma we may assume $0<\epsilon \leq \epsilon_{0}$ since the left-hand side of (44) is increasing in $\epsilon$.

We fix an auxiliary discrete subgroup $\Gamma \subset V$ of $\operatorname{rank} \operatorname{dim} V$ with the following property: for all $v \in V$ there exists $\gamma \in \Gamma$ with $|v-\gamma|<\epsilon$. Note that $\Gamma$ depends on $\epsilon$.

Let $\lambda \geq 1$ and $\varphi \in W$. For brevity we define $\mathcal{N}(\lambda)=\mathcal{N}_{\epsilon}\left(\lambda \varphi\left(\phi_{1}\left(U_{1}\right)\right)\right)$; recall that we wish to find a lower bound for the number of points in $(\gamma+\mathcal{N}(\lambda)) \cap \Omega$ in terms of $\lambda$ and uniform in $\varphi$ for some $\gamma$.

We claim

$$
B_{\epsilon \lambda}(0) \subset\left(B_{3 \lambda}(0) \cap \Gamma\right)+\mathcal{N}(\lambda),
$$

where the sum is the Minkowski sum, i.e. all sums of an element in the left set with an element in the right set. Indeed, say $x \in \mathbf{R}^{m_{2}}$ and $|x|<\epsilon \lambda$. Then $x / \lambda \in B_{\epsilon}(0) \subset B_{\epsilon_{0}}(0)$ and so $x / \lambda=\varphi\left(\phi_{1}\left(u_{1}\right)\right)+v$ for some $u_{1} \in U_{1}$ and $v \in V$ by (47). By our choice of $\Gamma$ there exists $\gamma \in \Gamma$ with $|\lambda v-\gamma|<\epsilon$. Hence $\left|x-\lambda \varphi\left(\phi_{1}\left(u_{1}\right)\right)-\gamma\right|=|\lambda v-\gamma|<\epsilon$ and so

$$
x \in \gamma+\mathcal{N}(\lambda) \quad \text { with } \gamma \in \Gamma .
$$


In order to deduce (48) it remains to show $|\gamma|<3 \lambda$. Because $|\lambda v-\gamma|<\epsilon$ we have $|\gamma|<\lambda|v|+\epsilon$. Now $|v|=\left|x / \lambda-\varphi\left(\phi_{1}\left(u_{1}\right)\right)\right| \leq|x| / \lambda+1$ by (46). We conclude $|\gamma| \leq|x|+\lambda+\epsilon<\epsilon \lambda+\lambda+\epsilon \leq 3 \lambda$ from $|x|<\epsilon \lambda$ and $\epsilon \leq 1 \leq \lambda$. Our claim (48) follows.

Counting points in $B_{\epsilon \lambda}(0) \cap \Omega$ is easier than in $\mathcal{N}(\lambda) \cap \Omega$ since $B_{\epsilon \lambda}(0)$ is convex. To make use of this observation we need

$$
\# B_{\epsilon \lambda}(0) \cap \Omega \leq \# B_{3 \lambda}(0) \cap \Gamma \cdot \max _{\gamma \in \Gamma} \#(\gamma+\mathcal{N}(\lambda)) \cap \Omega
$$

which follows from (48). So there exists $\gamma \in \Gamma$ such that

$$
\#\left(\lambda B_{\epsilon}(0)\right) \cap \Omega \leq \#\left(\lambda B_{3}(0)\right) \cap \Gamma \cdot \#(\gamma+\mathcal{N}(\lambda)) \cap \Omega .
$$

By geometry of numbers, more precisely the lower bound in (31), there exists $C_{23}>0$ which depends only on $\epsilon$ and $\Omega$ (but not on $\lambda$ ) such that $\#\left(\lambda B_{\epsilon}(0)\right) \cap \Omega \geq C_{23} \lambda^{\text {rk } \Omega}$ for all $\lambda \geq 1$. It follows from the upper bound in (31) that $\#\left(\lambda B_{3}(0)\right) \cap \Gamma \leq C_{24} \lambda^{\mathrm{rk}} \bar{\Gamma}$ for all $\lambda \geq 1$; here $C_{24}>0$ depends on $\Gamma$, and so ultimately on $\epsilon$, but not on $\lambda$. We conclude

$$
\frac{C_{23}}{C_{24}} \lambda^{\mathrm{rk} \Omega-\mathrm{rk} \Gamma} \leq \#(\gamma+\mathcal{N}(\lambda)) \cap \Omega
$$

from (49). The lemma follows with $C_{25}=C_{23} / C_{24}$ since $\operatorname{rk} \Omega-\operatorname{rk} \Gamma=$ $n_{1}+$ rk $\Omega-m_{2}$.

Lemma 16. Let $U \subset \mathbf{R}^{m}$ be open and let $\delta>0$ satisfy $\overline{B_{\delta}(0)} \subset U$. If $\phi: U \rightarrow \mathbf{R}^{m}$ is a smooth map and $M>0$ such that for all $x \in \overline{B_{\delta}(0)}$ the matrix $J_{x}(\phi)$ is invertible with $\left|J_{x}(\phi)^{-1}\right| \leq M$, then

$$
\mathcal{N}_{\delta /(8 M)}\left(\phi\left(B_{\delta / 2}(0)\right)\right) \subset \phi\left(B_{\delta}(0)\right) .
$$

Proof. By hypothesis and compactness there exists a bound $M^{\prime}>0$ for all second derivatives of all coordinates of $\phi$ on $\overline{B_{\delta}(0)}$. Thus for $x, x^{\prime}, x^{\prime \prime} \in$ $\overline{B_{\delta}(0)}$ we have

$$
\begin{aligned}
\left|J_{x}(\phi)^{-1}\left(J_{x^{\prime}}(\phi)-J_{x^{\prime \prime}}(\phi)\right)\right| & \leq\left|J_{x}(\phi)^{-1}\right|\left|J_{x^{\prime}}(\phi)-J_{x^{\prime \prime}}(\phi)\right| \\
& \leq C_{26} M M^{\prime}\left|x^{\prime}-x^{\prime \prime}\right|
\end{aligned}
$$

by the mean value theorem; here $C_{26}>0$ depends only on $m$.

We define

$$
r=\min \left\{\frac{1}{4 C_{26} M M^{\prime}}, \frac{\delta}{4}\right\} \leq \frac{\delta}{4} .
$$

So if $\left|x^{\prime}-x^{\prime \prime}\right| \leq 2 r$, then $\left|J_{x}(\phi)^{-1}\left(J_{x^{\prime}}(\phi)-J_{x^{\prime \prime}}(\phi)\right)\right| \leq 1 / 2$. Lemma 14 tells us that

$$
\text { if } \overline{B_{r}(x)} \subset B_{\delta}(0) \text { then } \overline{B_{r /(2 M)}(\phi(x))} \subset \phi\left(\overline{B_{r}(x)}\right) .
$$


Say $x \in B_{\delta / 2}(0), y_{0}=\phi(x)$, and $\left|y-y_{0}\right|<\delta /(8 M)$. To prove the lemma we must show $y \in \phi\left(B_{\delta}(0)\right)$. To do this, let

$$
N=1+\left[\frac{\delta}{4 r}\right] \geq 1
$$

and let $y_{0}, \ldots, y_{N}=y$ be equidistributed on the line segment between $y_{0}$ and $y$. Then

$$
\left|y_{k}-y_{k-1}\right|=\frac{\left|y-y_{0}\right|}{N}<\frac{\delta}{8 M N}<\frac{r}{2 M}
$$

since $N>\delta /(4 r)$.

We claim that for $0 \leq k \leq N$ there exists $x_{k} \in \mathbf{R}^{m}$ with

$$
\left|x_{k}\right|<\delta / 2+k r \leq \delta \quad \text { and } \phi\left(x_{k}\right)=y_{k} .
$$

The lemma then follows on taking $k=N$.

The inequality $\delta / 2+k r \leq \delta$ for $0 \leq k \leq N$ follows from $k r \leq N r \leq$ $r+\delta / 4 \leq \delta / 2$ where we used (52) and (50). We prove the rest of the claim by induction on $k$. If $k=0$ we can take $x_{0}=x$ so let us suppose $1 \leq k \leq N$ with $\left|x_{k-1}\right|<\delta / 2+(k-1) r$ and $\phi\left(x_{k-1}\right)=y_{k-1}$. By (53) we have $y_{k} \in B_{r /(2 M)}\left(y_{k-1}\right)=B_{r /(2 M)}\left(\phi\left(x_{k-1}\right)\right)$; furthermore, $\overline{B_{r}\left(x_{k-1}\right)} \subset B_{\delta}(0)$ follows from $r+\left|x_{k-1}\right|<r+\delta / 2+(k-1) r=\delta / 2+k r \leq \delta$. So by (51) we have $y_{k} \in \phi\left(\overline{B_{r}\left(x_{k-1}\right)}\right)$. Therefore we may choose $x_{k} \in \overline{\overline{B_{r}}\left(x_{k-1}\right)}$ with $\phi\left(x_{k}\right)=y_{k}$. Finally, our claim (54) and thus the lemma follow from $\left|x_{k}\right| \leq r+\left|x_{k-1}\right|<\delta / 2+k r$.

Lemma 17. Let $\varphi_{0} \in \operatorname{Mat}_{m_{2} m_{1}}(\mathbf{R})$ such that $J_{0}\left(\phi_{\varphi_{0}}\right)$ is invertible. There exist an open neighborhood $U \subset U_{1} \times U_{2}$ of 0 , a constant $\epsilon>0$, and an open neighborhood $W \subset \operatorname{Mat}_{m_{2} m_{1}}(\mathbf{R})$ of $\varphi_{0}$ such that if $\varphi \in W$ and $\lambda \geq 1$ then $\mathcal{N}_{\epsilon}\left(\phi_{\lambda \varphi}(U)\right) \subset \phi_{\lambda \varphi}\left(U_{1} \times U_{2}\right)$.

Proof. We fix $\delta>0$ and an open neighborhood $W \subset \operatorname{Mat}_{m_{2} m_{1}}(\mathbf{R})$ of $\varphi_{0}$ such that $\overline{B_{\delta}(0)} \subset U_{1} \times U_{2}$ and $J_{x}\left(\phi_{\varphi}\right)$ is invertible for all $x \in \overline{B_{\delta}(0)}$ and all $\varphi \in W$. Moreover, after shrinking $W$ if necessary, we may assume that there is an $M>0$ such that $\left|J_{x}\left(\phi_{\varphi}\right)^{-1}\right| \leq M$ for all $x \in \overline{B_{\delta}(0)}$ and all $\varphi \in W$.

Say $x \in \overline{B_{\delta}(0)}$ and $\varphi \in W$. For $\lambda>0$ the Jacobian matrix $J_{x}\left(\phi_{\lambda \varphi}\right)$ equals

$$
J_{x}\left(\phi_{\varphi}\right) S_{\lambda}
$$

where $S_{\lambda}$ is the $m_{2} \times m_{2}$ diagonal matrix with $\lambda$ 's in the first $n_{1}$ rows on the diagonal and 1's in the last $n_{2}$ rows on the diagonal. Hence $J_{x}\left(\phi_{\lambda \varphi}\right)$ is non-singular with inverse $S_{\lambda}^{-1} J_{x}\left(\phi_{\varphi}\right)^{-1}=S_{\lambda^{-1}} J_{x}\left(\phi_{\varphi}\right)^{-1}$.

Say $\lambda \geq 1$. It is not difficult to check $\left|S_{\lambda^{-1}}\right| \leq 1$, and so $\left|J_{x}\left(\phi_{\lambda \varphi}\right)^{-1}\right| \leq$ $\left|J_{x}\left(\phi_{\varphi}\right)^{-1}\right| \leq M$. From Lemma 16 applied to $\phi=\phi_{\lambda \varphi}$ we deduce

$$
\mathcal{N}_{\delta /(8 M)}\left(\phi_{\lambda \varphi}\left(B_{\delta / 2}(0)\right)\right) \subset \phi_{\lambda \varphi}\left(B_{\delta}(0)\right) \subset \phi_{\lambda \varphi}\left(U_{1} \times U_{2}\right) .
$$


This inclusion holds for all $\varphi \in W$ and $\lambda \geq 1$ and so the lemma follows with $\epsilon=\delta /(8 M)$ and $U=B_{\delta / 2}(0)$.

We are now ready to prove the main lemma of this section.

Proof of Lemma 13. By Lemma 17 there exist an open neighborhood $U \subset$ $U_{1} \times U_{2}$ of 0 , a constant $\epsilon>0$, and an open neighborhood $W \subset \operatorname{Mat}_{m_{2} m_{1}}(\mathbf{R})$ of $\varphi_{0}$ such that

$$
\phi_{\lambda \varphi}\left(U_{1} \times U_{2}\right) \supset \mathcal{N}_{\epsilon}\left(\phi_{\lambda \varphi}(U)\right) \text { for all } \varphi \in W \text { and all } \lambda \geq 1 \text {. }
$$

Without loss of generality we may assume $U=U_{1}^{\prime} \times U_{2}^{\prime}$ with $U_{i}^{\prime} \subset U_{i}$ open neighborhoods of 0 .

Soon we will apply Lemma 15 to $\varphi_{0}$ and $\phi_{1}$ (restricted to $U_{1}^{\prime}$ ) in order to find many points in $\left(\gamma+\mathcal{N}_{\epsilon}\left(\phi_{\lambda \varphi}(U)\right)\right) \cap \Omega$ for some $\epsilon>0$ and for $\gamma$ in an open subset of $\mathbf{R}^{m_{2}}$. But first we must verify that $J_{0}\left(\varphi_{0} \circ \phi_{1}\right)$ has rank $n_{1}$ : but this follows from the fact that the Jacobian matrix $J_{0}\left(\varphi_{0} \circ \phi_{1}\right)$ consists of the first $n_{1}$ columns of $J_{0}\left(\phi_{\varphi_{0}}\right)$. By possibly shrinking $W$, Lemma 15 applied to the $\epsilon$ from above implies that there exists a constant $C_{27}>0$ such that

$\#\left(\gamma+\mathcal{N}_{\epsilon}\left(\lambda \varphi\left(\phi_{1}\left(U_{1}^{\prime}\right)\right)\right)\right) \cap \Omega \geq C_{27} \lambda^{n_{1}+\mathrm{rk} \Omega-m_{2}} \quad$ for all $\varphi \in W$ and $\lambda \geq 1$ and all $\gamma$ in some non-empty open subset of $\mathbf{R}^{m_{2}}$.

Let $\varphi \in W$ and $\lambda \geq 1$. Because $U \supset U_{1}^{\prime} \times\{0\}$ and $\phi_{2}(0)=0$ we have

$$
\mathcal{N}_{\epsilon}\left(\phi_{\lambda \varphi}(U)\right) \supset \mathcal{N}_{\epsilon}\left(\lambda \varphi\left(\phi_{1}\left(U_{1}^{\prime}\right)\right)\right) \text {. }
$$

This together with (55) and (56) implies that

$$
\#\left(\gamma+\phi_{\lambda \varphi}\left(U_{1} \times U_{2}\right)\right) \cap \Omega \geq \#\left(\gamma+\mathcal{N}_{\epsilon}\left(\phi_{\lambda \varphi}(U)\right)\right) \cap \Omega \geq C_{27} \lambda^{n_{1}+\mathrm{rk} \Omega-m_{2}}
$$

holds for all $\gamma$ in some non-empty subset of $\mathbf{R}^{m_{2}}$.

Proof of Proposition 3. We choose smooth local parametrizations $\phi_{1}$ : $U_{1} \rightarrow M$ and $\phi_{2}: U_{2} \rightarrow N$ where $U_{1} \subset \mathbf{R}^{n_{1}}, U_{2} \subset \mathbf{R}^{n_{2}}$ are both open neighborhoods of 0 for $n_{1}=2 \operatorname{dim} M$ and $n_{2}=2 \operatorname{dim} N$; we also assume $\phi_{1}(0)=0, \phi_{2}(0)=0$. We identify $X$ with $\mathbf{R}^{m_{1}}$ and $Y$ with $\mathbf{R}^{m_{2}}$ where $m_{1}=2 \operatorname{dim} X$ and $m_{2}=2 \operatorname{dim} Y$. Any $\mathbf{C}$-linear map $X \rightarrow Y$ defines an $\mathbf{R}$-linear map $\mathbf{R}^{m_{1}} \rightarrow \mathbf{R}^{m_{2}}$ and hence can be identified with a matrix in $\operatorname{Mat}_{m_{2} m_{1}}(\mathbf{R})$. Let $\phi$ be as in the hypothesis, then $T_{0}(\phi)$ being an isomorphism implies that $J_{0}\left(\phi_{\varphi_{0}}\right)$ is invertible. The proposition follows from Lemma 13.

\section{A necessary condition for the vanishing of $v_{X}$}

All varieties in this section are defined over $\mathbf{C}$. Let $A$ and $B$ be abelian varieties and if not stated otherwise, $X$ is an irreducible closed subvariety of $A$ with $1 \leq r=\operatorname{dim} X \leq \operatorname{dim} B$. Recall that $v_{X}=v_{X}^{r}$ was introduced 
in Sect. 5, its definition requires a symmetric ample line bundle $\mathcal{L}_{B}$ on $B$ which we now also assume to be very ample.

The purpose of this section is to prove a necessary condition for the vanishing of $v_{X}$ at some element of $\operatorname{Hom}(A, B)_{\mathbf{R}}^{*}$. This is done in Proposition 4.

First we introduce some notation. If $s_{1}, \ldots, s_{k}$ are global sections of a line bundle on some variety, we define $Z\left(s_{1}, \ldots, s_{k}\right)$ to be the Zariski closed set of their common zeros.

We begin with several preliminary lemmas.

Lemma 18. Let $s_{1}, \ldots, s_{r}$ lie in $H^{0}\left(B, \mathscr{L}_{B}\right)$, the $\mathbf{C}$-vector space of global sections of $\mathcal{L}_{B}$. We assume that all irreducible components of $Z=$ $Z\left(s_{1}, \ldots, s_{r}\right)$ have dimension $\operatorname{dim} B-r$ and that $Y$ is such an irreducible component. For $\psi \in \operatorname{Hom}(A, B)$ there exists $V \subset B$ Zariski open and dense such that for all $v \in V(\mathbf{C})$ the intersection $(v+Z) \cap \psi(X)$ is finite and

$$
\left.\# \psi\right|_{X} ^{-1}(v+Y) \leq \operatorname{deg}\left(\left.\psi\right|_{X}\right) \cdot \#(v+Z) \cap \psi(X) .
$$

Proof. Let $Y^{\prime} \subset Z$ be any irreducible component and let $d_{Y^{\prime}}: X \times Y^{\prime} \rightarrow B$ be the morphism defined by $d_{Y^{\prime}}(p, y)=\psi(p)-y$.

Since $r+\operatorname{dim} Y^{\prime}=\operatorname{dim} B$, we may choose $V \subset B$ Zariski open and dense with $d_{Y^{\prime}}^{-1}(v)$ finite (possibly empty) for all $v \in V(\mathbf{C})$ by the theorem on the dimension of the fibres. By shrinking $V$ we may even assume that $d_{Y^{\prime}}^{-1}(v)$ is finite for all $v \in V(\mathbf{C})$ and all irreducible components $Y^{\prime}$ of $Z$. In particular,

$$
(v+Z) \cap \psi(X) \text { is finite for all } v \in V(\mathbf{C})
$$

and the first statement of the lemma follows.

We set $d=d_{Y}$. Let us assume first that $d$ is not dominant. Then after possibly shrinking $V \subset B$ we may assume that $V$ does not meet the image of $d$. In this case $\left.\psi\right|_{X} ^{-1}(v+Y)=\emptyset$ for all $v \in V(\mathbf{C})$ and the lemma follows. From now on we assume that $d$ is dominant.

The morphism $d$ is the composition of $(p, y) \mapsto(\psi(p),-y)$ and the addition morphism $B \times B \rightarrow B$. Now

$$
\begin{aligned}
\operatorname{dim} B=\operatorname{dim} d(X \times Y) & \leq \operatorname{dim} \psi(X) \times Y=\operatorname{dim} \psi(X)+\operatorname{dim} Y \\
& \leq r+\operatorname{dim} Y=\operatorname{dim} B
\end{aligned}
$$

and so equality must hold. We deduce $\operatorname{dim} \psi(X)=r$.

By the theorem on the dimension of the fibres there exists $U \subset X$ Zariski open and dense such that $\left.\psi\right|_{X} ^{-1}(\psi(p))$ is finite for all $p \in U(\mathbf{C})$. After shrinking $U$ we may assume

$$
\left.\# \psi\right|_{X} ^{-1}(\psi(p))=\operatorname{deg}\left(\left.\psi\right|_{X}\right) .
$$

We also have $\operatorname{dim}(X \backslash U) \times Y<r+\operatorname{dim} Y=\operatorname{dim} B$, so $d((X \backslash U) \times Y) \subset B$ is not Zariski dense. After possibly shrinking $V$ a final time we may assume

$$
V \cap d((X \backslash U) \times Y)=\emptyset .
$$


We now show (57), so say $v \in V(\mathbf{C})$. If $q \in(v+Y(\mathbf{C})) \cap \psi(X)$, then $q=\psi(p)$ for some $p \in X(\mathbf{C})$ with $\psi(p)-v \in Y(\mathbf{C})$. We deduce $p \in U(\mathbf{C})$ from (60). This together with (59) implies that the inner sum on the right of

$$
\left.\# \psi\right|_{X} ^{-1}(v+Y)=\sum_{q \in(v+Y(\mathbf{C})) \cap \psi(X)} \sum_{\substack{p \in X(\mathbf{C}) \\ \psi(p)=q}} 1
$$

equals $\operatorname{deg}\left(\left.\psi\right|_{X}\right)$. The outer sum is also over a finite set by (58). Thus the lemma follows.

Lemma 19. In the same situation as in the preceding lemma we have

$$
\left.\# \psi\right|_{X} ^{-1}(v+Y) \leq v_{X}(\psi) \quad \text { for } v \in V(\mathbf{C}) .
$$

Proof. If $\operatorname{dim} \psi(X)<r$, then $\operatorname{deg}\left(\left.\psi\right|_{X}\right)=0$; thus by the last assertion of Lemma 18 the left-hand side of $(61)$ is zero. The lemma follows in this case. Hence we will assume $\operatorname{dim} \psi(X)=r$.

Say $v \in V(\mathbf{C})$. Recall that $t_{-v}: B \rightarrow B$ is the translation by $-v$ morphism. We may rewrite $v+Z$ as $Z\left(s_{1} \circ t_{-v}, \ldots, s_{r} \circ t_{-v}\right)$, here $s_{i} \circ t_{-v}$ is a global section of $t_{-v}{ }^{*} \mathcal{L}_{B}$. By Lemma 18 we get

$$
\begin{aligned}
\left.\# \psi\right|_{X} ^{-1}(v+Y) & \leq \operatorname{deg}\left(\left.\psi\right|_{X}\right) \cdot \# Z\left(s_{1} \circ t_{-v}, \ldots, s_{r} \circ t_{-v}\right) \cap \psi(X) \\
& =\operatorname{deg}\left(\left.\psi\right|_{X}\right) \cdot \# Z\left(\left.s_{1} \circ t_{-v}\right|_{\psi(X)}, \ldots,\left.s_{r} \circ t_{-v}\right|_{\psi(X)}\right),
\end{aligned}
$$

where $\left.s_{i} \circ t_{-v}\right|_{\psi(X)}$ is a global section of $\left.t_{-v}\right|_{\psi(X)} ^{*} \mathcal{L}_{B} \in \operatorname{Pic}(\psi(X))$.

Since $\operatorname{dim} \psi(X)=r$ and because $Z\left(\left.s_{1} \circ t_{-v}\right|_{\psi(X)}, \ldots,\left.s_{r} \circ t_{-v}\right|_{\psi(X)}\right)$ is finite the $r$ sections $\left.s_{i} \circ t_{-v}\right|_{\psi(X)}$ intersect properly on $\psi(X)$. Intersection theory, cf. [11, Example 7.1.10(a), p. 123], gives

$$
\# Z\left(\left.s_{1} \circ t_{-v}\right|_{\psi(X)}, \ldots,\left.s_{r} \circ t_{-v}\right|_{\psi(X)}\right) \leq\left(c_{1}\left(\left.t_{-v}\right|_{\psi(X)} ^{*} \mathcal{L}_{B}\right)^{r}[\psi(X)]\right) .
$$

With (62) we obtain

$$
\left.\# \psi\right|_{X} ^{-1}(v+Y) \leq \operatorname{deg}\left(\left.\psi\right|_{X}\right)\left(c_{1}\left(\left.t_{-v}\right|_{\psi(X)} ^{*} \mathcal{L}_{B}\right)^{r}[\psi(X)]\right) .
$$

Now $t_{-v}{ }^{*} \mathcal{L}_{B}=\mathscr{L}_{B} \otimes \mathcal{M}$ with $\mathcal{M}$ algebraically equivalent to zero. And so $\left.t_{-v}\right|_{\psi(X)} ^{*} \mathcal{L}_{B}=\left.\left.\mathcal{L}_{B}\right|_{\psi(X)} \otimes \mathcal{M}\right|_{\psi(X)}$ where $\left.\mathcal{M}\right|_{\psi(X)}$ is also algebraically equivalent to zero. It follows that $c_{1}\left(\left.t_{-v}\right|_{\psi(X)} ^{*} \mathscr{L}_{B}\right)=c_{1}\left(\left.\mathscr{L}_{B}\right|_{\psi(X)}\right)$, thus

$$
\left.\# \psi\right|_{X} ^{-1}(v+Y) \leq \operatorname{deg}\left(\left.\psi\right|_{X}\right)\left(c_{1}\left(\left.\mathcal{L}_{B}\right|_{\psi(X)}\right)^{r}[\psi(X)]\right) .
$$

But by Lemma 6(iii) the right-hand side is $v_{X}(\psi)$ and the lemma follows.

From now on we assume that

$$
0 \text { is a non-singular point of } X \text {. }
$$


All references to a topology, for example on $A(\mathbf{C})$, will mean the complex topology unless stated otherwise with the exception that irreducibility always refers to the Zariski topology.

As in Sect. 6 we regard $A(\mathbf{C})$ as a complex Lie group and let $\exp _{A}$ : $T(A) \rightarrow A(\mathbf{C})$ denote the exponential map. Then $\exp _{A}$ is an open holomorphic map between complex manifolds and a homomorphism of groups. Furthermore, the associated map between tangent spaces at the unit element is $T_{0}(T(A))=T(A) \rightarrow T(A)$ is the identity. By the inverse mapping theorem, there exists $U_{A} \subset A(\mathbf{C})$ open and containing 0 and a biholomorphic map $l_{A}: U_{A} \rightarrow l_{A}\left(U_{A}\right) \subset T(A)$ which is locally an inverse of $\exp _{A}$. Naturally, we may carry out the same constructions for $B$ and obtain $U_{B}$ and $l_{B}$.

After possibly shrinking $U_{A}$ and because of (63) we may assume that $U_{A} \cap \operatorname{Sing}(X)=\emptyset$. Then $X(\mathbf{C}) \cap U_{A}$ is a complex submanifold of $U_{A}$. Furthermore,

$$
M=l_{A}\left(X(\mathbf{C}) \cap U_{A}\right)
$$

is a complex submanifold of $T(A)$. We may identify the tangent space of a submanifold with a subspace of the tangent space of the ambient manifold. Hence we take $T_{0}(M)$ as a vector subspace of $T(A)$.

Recall that any element $\psi \in \operatorname{Hom}(A, B)$ defines a $\mathbf{C}$-linear map $T(\psi)$ between the tangent spaces at 0 of $A$ and $B$. Moreover,

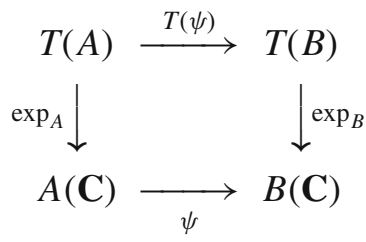

commutes. We also defined $T(\psi)$ for $\psi \in \operatorname{Hom}(A, B)_{\mathbf{R}}$ in Sect. 3. Just as in the case of a power of an elliptic curve covered in Sect. 6 such a $\psi$ need not be a homomorphism between abelian varieties even after multiplication with a non-zero scalar. On the other hand, $T(\psi): T(A) \rightarrow T(B)$ considered as a linear map is clearly holomorphic and its restriction to $M$ defines a holomorphic map $M \rightarrow T(B)$ between complex manifolds.

In order to prove Proposition 4, the main result of this section, we need to construct an auxiliary subvariety of $A$ whose tangent space at 0 lies in general position.

Lemma 20. Let $T \subset T(B)$ be a $\mathbf{C}$-vector subspace. For an integer $t$ with $0 \leq t \leq \operatorname{dim} T$ there exist global sections $s_{1}, \ldots, s_{t}$ of $\mathcal{L}_{B}$ such that

(i) 0 is a non-singular point of $Z=Z\left(s_{1}, \ldots, s_{t}\right)$, hence a neighborhood of 0 in $Z(\mathbf{C})$ is a complex submanifold of $B(\mathbf{C})$,

(ii) $\operatorname{dim} T \cap T_{0}(Z)=\operatorname{dim} T-t$, and

(iii) all irreducible components of $Z$ have dimension $\operatorname{dim} B-t$. 
Proof. We prove the lemma by induction on $t$. The case $t=0$ being trivial, we assume $1 \leq t \leq \operatorname{dim} T$. We choose $s_{1}, \ldots, s_{t-1} \in H^{0}\left(B, \mathcal{L}_{B}\right)$ satisfying the assertion of the lemma for $t-1$.

By the convention made at the beginning of this section $\mathcal{L}_{B}$ is very ample. Hence, each choice of an (ordered) basis of $H^{0}\left(B, \mathcal{L}_{B}\right)$, of cardinality $n+1$ say, determines a closed immersion $\iota: B \rightarrow \mathbf{P}^{n}$. By choosing a suitable basis $s_{0}^{\prime}, \ldots, s_{n}^{\prime}$ of $H^{0}\left(B, \mathcal{L}_{B}\right)$ we may assume that $\iota(0)=[1$ : $0: \cdots: 0]$. Let $Z\left(s_{1}, \ldots, s_{t-1}\right)$ have irreducible components $Y_{1}, \ldots, Y_{m}$. Since $\operatorname{dim} Y_{i}=\operatorname{dim} B-(t-1) \geq \operatorname{dim} B-(\operatorname{dim} T-1) \geq 1$ we can choose $p_{i} \in Y_{i}(\mathbf{C}) \backslash\{0\}$. Hence $\iota\left(p_{i}\right) \neq \iota(0)$ and we see that for each $i$ at least one among $s_{1}^{\prime}\left(p_{i}\right), \ldots, s_{n}^{\prime}\left(p_{i}\right)$ does not vanish. We conclude that for generic $\left(\lambda_{1}, \ldots, \lambda_{n}\right) \in \mathbf{C}^{n}$ the global section

$$
s_{t}=\lambda_{1} s_{1}^{\prime}+\cdots+\lambda_{n} s_{n}^{\prime}
$$

vanishes on 0 but on none of the $p_{i}$. Hence $s_{1}, \ldots, s_{t-1}, s_{t}$ cut out a Zariski closed subset of $B$ containing 0 whose irreducible components have dimension $\operatorname{dim} B-t$. Furthermore, if $\left(\lambda_{1}, \ldots, \lambda_{n}\right)$ is generic the origin is a non-singular point of $Z(\mathbf{C})$ and assertion (ii) holds.

We are now ready to prove Proposition 4. It generalizes Lemma 9 and relies on the counting result developed in Sect. 7.

Proposition 4. In the notation introduced above let $\varphi_{0} \in \operatorname{Hom}(A, B)_{\mathbf{R}}$ such that

$$
\left.T\left(\varphi_{0}\right)\right|_{T_{0}(M)}=T_{0}\left(\left.T\left(\varphi_{0}\right)\right|_{M}\right): T_{0}(M) \rightarrow T(B)
$$

has rank $r=\operatorname{dim} X$, then

$$
v_{X}\left(\varphi_{0}\right)>0
$$

Proof. Consider $T \subset T(B)$, the image of $T_{0}(M)$ under $T\left(\varphi_{0}\right)$, it is a $\mathbf{C}$-vector space of dimension $r$ by hypothesis. We apply Lemma 20 with $t=r$ to $T$ and obtain global sections $s_{1}, \ldots, s_{r}$ of $\mathcal{L}_{B}$ and a Zariski closed $Z \subset B$ satisfying the stated properties.

Since $0 \in Z(\mathbf{C})$ is a non-singular point there exists a unique irreducible component $Y \subset Z$ of dimension $\operatorname{dim} B-r$ containing 0 as a non-singular point. After shrinking $U_{B}$ if necessary we may assume that $Y(\mathbf{C}) \cap U_{B}$ is a complex submanifold of $B(\mathbf{C})$. Then $N=l_{B}\left(Y(\mathbf{C}) \cap U_{B}\right)$ is a complex submanifold of $T(B)$. By the second part of Lemma 20 we have

$$
T \cap T_{0}(N)=0 .
$$

Let $\phi: M \times N \rightarrow T(B)$ be the holomorphic map defined by $\phi(x, y)=$ $T\left(\varphi_{0}\right)(x)-y$. Then $T_{0}(\phi): T_{0}(M \times N)=T_{0}(M) \times T_{0}(N) \rightarrow T_{0}(T(B))=$ $T(B)$ is given by $T_{0}(\phi)(u, v)=T\left(\varphi_{0}\right)(u)-v$ for $u \in T_{0}(M)$ and $v \in$ $T_{0}(N)$. We claim that $T_{0}(\phi)$ is an isomorphism. Indeed, since $\operatorname{dim} M+$ $\operatorname{dim} N=\operatorname{dim} B$ it suffices to prove injectivity. Now if $T\left(\varphi_{0}\right)(u)=v$, then 
$v=0$ by (65). Furthermore, $\left.T_{0}\left(\varphi_{0}\right)\right|_{T_{0}(M)}$ is injective since it has rank $r$ by hypothesis. Hence $u=0$ and our claim follows.

The kernel $\Omega$ of $\exp _{B}$ is a discrete subgroup of $T(B)$ with rank $2 \operatorname{dim} B$. We apply Proposition 3 and obtain a constant $C_{28}>0$, an open neighborhood $W \subset \operatorname{Hom}(T(A), T(B))$ of $T\left(\varphi_{0}\right)$, and for each $\varphi \in \operatorname{Hom}(A, B)_{\mathbf{R}}$ and each $\lambda \geq 1$ with $T(\varphi) \in W$ an open non-empty set $S(\varphi, \lambda) \subset T(B)$ such that

$$
\#(\gamma+\lambda T(\varphi)(M)-N) \cap \Omega \geq C_{28} \lambda^{2 r}
$$

for all $\gamma \in S(\varphi, \lambda)$. The constant $C_{28}$ is independent of $\varphi$ and $\lambda$.

Say now $\varphi \in \operatorname{Hom}(A, B)_{\mathbf{Q}}$ such that $T(\varphi) \in W$. We choose a positive integer $\lambda$ with $\lambda \varphi \in \operatorname{Hom}(A, B)$. Having fixed $\varphi$ and $\lambda$ let $\gamma \in S(\varphi, \lambda)$ be arbitrary. There exist $\omega_{1}, \ldots, \omega_{\Delta} \in \Omega$ which are distinct, $x_{1}, \ldots, x_{\Delta} \in M$, and $y_{1}, \ldots, y_{\Delta} \in N$ such that

$$
\omega_{i}=\gamma+\lambda T(\varphi)\left(x_{i}\right)-y_{i} \text { and } \Delta \geq C_{28} \lambda^{2 r} .
$$

We claim that the

$$
\exp _{A}\left(x_{1}\right), \ldots, \exp _{A}\left(x_{\Delta}\right) \text { are distinct. }
$$

Indeed, since $\exp _{A}$ is injective on $M \subset l_{A}\left(U_{A}\right)$, it suffices to show that the $x_{i}$ are distinct. Let us assume $x_{i}=x_{j}$, then $\omega_{i}+y_{i}=\omega_{j}+y_{j}$ by (66). Hence $y_{i}-y_{j} \in \Omega$ and thus $\exp _{B}\left(y_{i}\right)=\exp _{B}\left(y_{j}\right)$. But $y_{i}, y_{j} \in N$ and $\exp _{B}$ is injective on $N \subset l_{B}\left(U_{B}\right)$, so $y_{i}=y_{j}$. This implies $\omega_{i}=\omega_{j}$ and so $i=j$.

On applying $\exp _{B}$ to (66) we obtain

$0=\exp _{B}\left(\gamma+\lambda T(\varphi)\left(x_{i}\right)-y_{i}\right)=\exp _{B}(\gamma)+\exp _{B}\left(T(\lambda \varphi)\left(x_{i}\right)\right)-\exp _{B}\left(y_{i}\right)$.

We recall that $\psi=\lambda \varphi \in \operatorname{Hom}(A, B)$ by our choice of $\lambda$. Therefore $\exp _{B} \circ$ $T(\psi)=\psi \circ \exp _{A}$ by (64) giving $\psi\left(\exp _{A}\left(x_{i}\right)\right)=\exp _{B}(-\gamma)+\exp _{B}\left(y_{i}\right)$. Now $y_{i} \in N$, so $\exp _{B}\left(y_{i}\right) \in Y(\mathbf{C})$ hence

$$
\psi\left(\exp _{A}\left(x_{i}\right)\right) \in \exp _{B}(-\gamma)+Y(\mathbf{C}) .
$$

Since the image of $x_{i} \in M$ under $\exp _{A}$ is in $X(\mathbf{C})$ it follows that the $\exp _{A}\left(x_{i}\right)$ are in the fibre $\left.\psi\right|_{X} ^{-1}\left(\exp _{B}(-\gamma)+Y\right)$. By the arguments around (67) they are also distinct. We conclude

$$
\left.\# \psi\right|_{X} ^{-1}\left(\exp _{B}(-\gamma)+Y\right) \geq \Delta \geq C_{28} \lambda^{2 r},
$$

and this inequality holds for all $\gamma \in S(\varphi, \lambda)$.

We would like to bound the left-hand side of (68) from above in terms of $v_{X}(\psi)$ using Lemma 19. Let $V \subset B$ be Zariski open and non-empty as in this lemma. Recall that $\exp _{B}$ is an open map. So the image of $-S(\varphi, \lambda)$ under $\exp _{B}$ is open in $B(\mathbf{C})$ and thus Zariski dense in $B$ by (35). Hence there is $\gamma \in S(\varphi, \lambda)$ such that $\exp _{B}(-\gamma) \in V(\mathbf{C})$. So inequality (61) holds with $v=\exp _{B}(-\gamma)$. Together with (68) we obtain $v_{X}(\psi) \geq C_{28} \lambda^{2 r}$. Now 
$\psi=\lambda \varphi$ and $v_{X}$ is homogeneous of degree $2 r$ so $\lambda^{2 r} v_{X}(\varphi) \geq C_{28} \lambda^{2 r}$. Cancelling $\lambda$ gives

$$
v_{X}(\varphi) \geq C_{28} .
$$

Inequality (69) holds for any $\varphi \in \operatorname{Hom}(A, B)_{\mathbf{Q}}$ with $T(\varphi) \in W$. This set is dense in the set of all $\varphi \in \operatorname{Hom}(A, B)_{\mathbf{R}}$ with $T(\varphi) \in W$. By continuity of $v_{X}$ and since $T\left(\varphi_{0}\right) \in W$ we conclude

$$
v_{X}\left(\varphi_{0}\right) \geq C_{28} .
$$

The proposition follows since $C_{28}>0$.

This proposition also holds true if the line bundle $\mathcal{L}_{B}$ in the definition of $v_{X}$ is merely symmetric and ample. Indeed, passing to a positive power of $\mathcal{L}_{B}$ corresponds to multiplying $v_{X}$ by a positive integer.

\section{Applying Ax's theorem}

All varieties in this section are defined over $\mathbf{C}$. Let $A$ and $B$ be abelian varieties. If not stated otherwise, let $X$ be an irreducible closed subvariety of $A$ with $1 \leq r=\operatorname{dim} X \leq \operatorname{dim} B$. In order to make sense of $v_{X}$ we must fix an ample and symmetric line bundle which we also assume to be very ample in accordance with Sect. 8.

In this section we will use Ax's theorem, cf. Sect. 6, to show that $v_{X}$ does not vanish on $\operatorname{Hom}(A, B)_{\mathbf{R}}^{*}$ as soon as $X^{\text {oa, }[\operatorname{dim} B]} \neq \emptyset$. In fact, we will prove this for irreducible subvarieties of $X$ meeting $X^{\mathrm{oa},[\mathrm{dim} B]}$.

Lemma 21. Assume that there exists $\varphi_{0} \in \operatorname{Hom}(A, B)_{\mathbf{R}}^{*}$ such that $v_{X}\left(\varphi_{0}\right)=0$. Then there is a non-empty subset $V \subset X(\mathbf{C})$ which is open in the complex topology such that for any $p \in V$ there exists an algebraic subgroup $H \subset A$ with

$$
\operatorname{dim}_{p} X \cap(p+H) \geq \max \{1, \operatorname{dim} B+\operatorname{dim} H-\operatorname{dim} A+1\} .
$$

In particular, $p \notin X^{\mathrm{oa},[\operatorname{dim} B]}$ for all $p \in V$.

Proof. Unless stated otherwise, all references to topology in this proof will mean the complex topology. We also use some notation introduced in Sect. 8.

By Lemma 6(ii) it suffices to prove the lemma for any translate of $X$. Hence we may assume that $X$ contains 0 as a non-singular point, i.e. it satisfies (63). Recall that $\exp _{A}$ is locally biholomorphic at 0 . So there is a complex submanifold $M \subset T(A)$ mapped by $\exp _{A}$ biholomorphically to an open neighborhood of 0 in $X(\mathbf{C})$ such that this neighborhood is a complex submanifold of $A(\mathbf{C})$. Clearly, we may assume $0 \in M$.

By Proposition 4, the rank of $T_{0}\left(\left.T\left(\varphi_{0}\right)\right|_{M}\right)$ is at most $r-1$. Moreover, as in the proof of Lemma 11, the same holds for the rank of $T_{z}\left(\left.T\left(\varphi_{0}\right)\right|_{M}\right)$ for all $z \in M$ after possibly shrinking $M$. 
By Lemma 10 there is a non-empty open subset $U \subset M$ such that the fibre $\left.T\left(\varphi_{0}\right)\right|_{U} ^{-1}\left(T\left(\varphi_{0}\right)(z)\right)$ is a complex submanifold of $T(A)$ of positive dimension for arbitrary $z \in U$. Therefore, so is $(U-z) \cap \operatorname{ker} T\left(\varphi_{0}\right)$. We may assume that $\exp _{A}$ is biholomorphic when restricted to $U-z$. Let $G$ be the connected analytic group $\exp _{A}\left(\operatorname{ker} T\left(\varphi_{0}\right)\right) \subset A(\mathbf{C})$. Then $\exp _{A}\left((U-z) \cap \operatorname{ker} T\left(\varphi_{0}\right)\right) \subset G$ contains a connected complex submanifold $K \subset A(\mathbf{C})$ with $0 \in K$ and $\operatorname{dim} K \geq 1$. Certainly, $K$ is also contained in $X(\mathbf{C})-p$ where $p=\exp _{A}(z)$.

Let $Z$ be the Zariski closure of $K$ in $A$ and let $H$ be the minimal algebraic subgroup containing $Z$. We have the inclusions $p+Z \subset X$, $p+Z \subset p+H$, and $p \in p+Z$ and it follows from [12, theorem on p. 168] that $Z$ is irreducible in the Zariski topology. Hence $\operatorname{dim}_{p} X \cap$ $(p+H) \geq \operatorname{dim} Z$. Ax's theorem implies $\operatorname{dim} H \leq \operatorname{dim} Z+\operatorname{dim} G-$ $\operatorname{dim} K \leq \operatorname{dim} Z+\operatorname{dim} G-1$. By hypothesis $\operatorname{ker} T\left(\varphi_{0}\right)$ has dimension $\operatorname{dim} A-\operatorname{dim} B$ and this is also the dimension of $G$. Hence $\operatorname{dim} H \leq$ $\operatorname{dim} Z+\operatorname{dim} A-\operatorname{dim} B-1$. But $\operatorname{dim} Z \geq 1, \operatorname{sod} \operatorname{dim} Z \geq \max \{1, \operatorname{dim} B+$ $\operatorname{dim} H-\operatorname{dim} A+1\}$. The lemma follows with $V=\exp _{A}(U)$ which is open in $X(\mathbf{C})$.

Unfortunately, the algebraic subgroup $H$ in the lemma above may depend on $p$. In Lemma 24 we will show that one can choose $H$ independent of $p$, but first we need two preparatory lemmas.

Lemma 22. Let $H \subset A$ be an algebraic subgroup and let $k \in \mathbf{Z}$. The set

$$
Z_{H}=\left\{p \in X(\mathbf{C}) ; \operatorname{dim}_{p} X \cap(p+H) \geq k\right\}
$$

is Zariski closed in $X$.

Proof. Say $\psi: A \rightarrow A / H$ and let

$$
Z_{H}^{\prime}=\left\{p \in X(\mathbf{C}) ;\left.\operatorname{dim}_{p} \psi\right|_{X} ^{-1}(\psi(p)) \geq k\right\} .
$$

By the theorem on semi-continuity of Chevalley, cf. [10, second theorem on p. 228], $Z_{H}^{\prime}$ is Zariski closed in $X$. We claim $Z_{H}^{\prime}=Z_{H}$. If $p \in Z_{H}^{\prime}$, then there exists an irreducible closed subvariety $Z \subset X$ of dimension at least $k$, with $p \in Z(\mathbf{C})$, and which is contained in the fibre of $\psi(p)$. Then $Z-p$ is contained in $H$, so $p \in Z_{H}$. Conversely, if $p \in Z_{H}$ is contained in an irreducible $Z$ with $Z \subset X \cap(p+H)$ and $\operatorname{dim} Z \geq k$, then $\left.Z \subset \psi\right|_{X} ^{-1}(\psi(p))$ and so $p \in Z_{H}^{\prime}$.

Lemma 23. Let $Z_{1}, Z_{2}, \ldots$ be countably many Zariski closed subsets of $X$ such that $\bigcup_{i} Z_{i}(\mathbf{C})$ contains a non-empty set which is open in the complex topology of $X(\mathbf{C})$. Then there exists $i$ with $X=Z_{i}$.

Proof. By the Baire category theorem we may assume that already one $Z_{1}(\mathbf{C})$ contains a non-empty subset of $X(\mathbf{C})$ which is open in the complex topology. The lemma then follows from (35). 
Lemma 24. Assume that there exists $\varphi_{0} \in \operatorname{Hom}(A, B)_{\mathbf{R}}^{*}$ such that $v_{X}\left(\varphi_{0}\right)=0$. Then there exists an algebraic subgroup $H \subset A$ with

$$
\operatorname{dim}_{p} X \cap(p+H) \geq \max \{1, \operatorname{dim} B+\operatorname{dim} H-\operatorname{dim} A+1\}
$$

for all $p \in X(\mathbf{C})$.

Proof. It is well-known that the abelian variety $A$ has only countably many algebraic subgroups, say $H_{1}, H_{2}, \ldots$ The set $Z_{i}$ of all $p \in X(\mathbf{C})$ such that $\operatorname{dim}_{p} X \cap\left(p+H_{i}\right) \geq \max \left\{1, \operatorname{dim} B+\operatorname{dim} H_{i}-\operatorname{dim} A+1\right\}$ is Zariski closed in $X$ by Lemma 22. By Lemma 21 the countable union over all $Z_{i}(\mathbf{C})$ contains a non-empty set which is open in the complex topology. We apply Lemma 23 to the $Z_{i}$ and obtain $X=Z_{i}$ for some $i$. The lemma follows with $H=H_{i}$.

Proposition 5. Let $Y$ be an irreducible closed subvariety of $X$ with $\operatorname{dim} Y \geq 1$. If $Y(\mathbf{C}) \cap X^{\text {oa, }[\operatorname{dim} B]} \neq \emptyset$ then $v_{Y}(\varphi)>0$ for all $\varphi \in \operatorname{Hom}(A, B)_{\mathbf{R}}^{*}$ or in other words (21) holds with $X$ replaced by $Y$.

Proof. Let $Y$ be an irreducible closed subvariety of $X$ with $\operatorname{dim} Y \geq 1$ such that $v_{Y}$ vanishes at some element of $\operatorname{Hom}(A, B)_{\mathbf{R}}^{*}$. Lemma 24 applied to $Y$ implies that there exists an algebraic subgroup $H$ of $A$ such that $\operatorname{dim}_{p} Y \cap(p+H) \geq \max \{1, \operatorname{dim} B+\operatorname{dim} H-\operatorname{dim} A+1\}$ for all $p \in Y(\mathbf{C})$. Therefore, $Y(\mathbf{C}) \cap X^{\text {oa, }[\mathrm{dim} B]}=\emptyset$ as desired.

\section{Completion of the proof of the bounded height theorem and the corollaries}

Let $A$ and $X$ be as in the bounded height theorem and fix an ample symmetric line bundle with $\hat{h}$ the associated Néron-Tate height on $A(\overline{\mathbf{Q}})$.

In order to prove our theorem it suffices to consider $s \geq \operatorname{dim} X$ since $X^{\mathrm{oa},[s]}=\emptyset$ otherwise. Also, we may assume $\operatorname{dim} X \geq 1$. For brevity we write $\Sigma=X^{\mathrm{oa},[s]}(\overline{\mathbf{Q}})$.

As in the end of Sect. 5 we may fix once and for all abelian varieties $B_{1}, \ldots, B_{n}$ with $\operatorname{dim} B_{i} \geq s$ such that for any $p \in A^{[s]}$ there exist an $1 \leq i \leq n$ and a surjective homomorphism $\psi: A \rightarrow B_{i}$ with $\psi(p)=0$.

Lemma 25. Say there exist a proper subset $\Sigma_{1} \subsetneq \Sigma$ and $\epsilon_{1}>0$ such that $\hat{h}$ is bounded on $\Sigma_{1} \cap \mathcal{C}\left(A^{[s]}, \epsilon_{1}\right)$. Then there exist a subset $\Sigma_{2} \subset \Sigma$ containing $\Sigma_{1}$ and $\epsilon_{2}>0$ such that $\hat{h}$ is bounded on $\Sigma_{2} \cap \mathcal{C}\left(A^{[s]}, \epsilon_{2}\right)$ and such that $\overline{\Sigma \backslash \Sigma_{2}} \subsetneq \overline{\Sigma \backslash \Sigma_{1}}$ for the Zariski closures.

Proof. By hypothesis $\Sigma \backslash \Sigma_{1}$ is non-empty, so $\overline{\Sigma \backslash \Sigma_{1}}$ has an irreducible component $Y \subset X$ with $Y(\overline{\mathbf{Q}}) \cap \Sigma \neq \emptyset$. We can decompose $\overline{\Sigma \backslash \Sigma_{1}}=$ $Y \cup Z$ with $Z$ Zariski closed and $Y \not \subset Z$. If $\operatorname{dim} Y \geq 1$ we note $Y(\mathbf{C}) \cap$ $X^{\text {oa, }\left[\mathrm{dim} B_{i}\right]} \supset Y(\overline{\mathbf{Q}}) \cap \Sigma \neq \varnothing$ and apply Proposition 5 to conclude that (21) holds with $X$ replaced by $Y$ and for all $B=B_{i}$. In particular, the hypothesis 
of Proposition 1 is satisfied with $X$ replaced by $Y$ and for all $B=B_{i}$. For a fixed $B_{i}$ there exist $U \subset Y$ Zariski open and dense, $\epsilon_{2}>0$, and $C_{29} \in \mathbf{R}$ such that: if $p \in U(\overline{\mathbf{Q}})$ with $p=a+b$ where $\psi(a)=0$ for some surjective homomorphism $\psi: A \rightarrow B_{i}$ and $\hat{h}(b) \leq \epsilon_{2}(1+\hat{h}(a))$ then $\hat{h}(p) \leq C_{29}$. Since there are only finitely many $B_{i}$ we may choose $U, \epsilon_{2}$, and $C_{29}$ independent of $i$. In the case $\operatorname{dim} Y=0$ all of this holds trivially.

By the remark made before this lemma we conclude that $\hat{h}$ is bounded from above by $C_{29}$ on $U(\overline{\mathbf{Q}}) \cap \mathcal{C}\left(A^{[s]}, \epsilon_{2}\right)$. Certainly we may assume $\epsilon_{2} \leq \epsilon_{1}$. We set $\Sigma_{2}=\Sigma_{1} \cup(U(\overline{\mathbf{Q}}) \cap \Sigma)$ and immediately deduce that the height is bounded from above on $\Sigma_{2} \cap \mathcal{C}\left(A^{[s]}, \epsilon_{2}\right)$.

Clearly, $\overline{\Sigma \backslash \Sigma_{2}} \subset \overline{\Sigma \backslash \Sigma_{1}}$ and to prove the last assertion it suffices to shows that the inclusion is strict. Now $\Sigma \backslash \Sigma_{2} \subset(Y \backslash U)(\overline{\mathbf{Q}}) \cup Z(\overline{\mathbf{Q}})$ by construction and so $\overline{\Sigma \backslash \Sigma_{2}} \subset(Y \backslash U) \cup Z$. But $Y \subset \overline{\Sigma \backslash \Sigma_{1}}$ is not contained $(Y \backslash U) \cup Z$, so the lemma follows.

Proof of the bounded height theorem. Recall that $\Sigma=X^{\mathrm{oa},[s]}(\overline{\mathbf{Q}})$. We set $\Sigma_{0}=\emptyset$ and $\epsilon_{0}=1$. Let $k \geq 1$. By induction we assume $\Sigma_{k-1}$ is a subset of $\Sigma$ such that $\Sigma_{k-1} \cap \mathcal{C}\left(A^{[\bar{s}]}, \epsilon_{k-1}\right)$ has bounded height for some $\epsilon_{k-1}>0$. If $\Sigma_{k-1}=\Sigma$ we are done, so let us assume $\Sigma_{k-1} \subsetneq \Sigma$. In this case we apply Lemma 25 to find a subset $\Sigma_{k} \subset \Sigma$ containing $\Sigma_{k-1}$ and $\epsilon_{k}>0$ such that $\hat{h}$ is bounded on $\Sigma_{k} \cap \mathcal{C}\left(A^{[s]}, \epsilon_{k}\right)$ and

$$
X \supset \overline{\Sigma \backslash \Sigma_{0}} \supsetneq \overline{\Sigma \backslash \Sigma_{1}} \supsetneq \cdots \supsetneq \overline{\Sigma \backslash \Sigma_{k}} \text {. }
$$

But $X$ is a noetherian topological space and therefore it satisfies the descending chain condition for Zariski closed sets. In our situation this means that $\Sigma_{k}=\Sigma$ for some $k$, the theorem follows.

Finally, we turn to the proof of Corollaries 1 and 2.

In her recent $\mathrm{Ph} . \mathrm{D}$. thesis, Carrizosa proved the following relative Lehmer-type height lower bound.

Theorem (Carrizosa). Let $A$ be an abelian variety with complex multiplication and $\hat{h}$ the Néron-Tate height on $A(\overline{\mathbf{Q}})$ associated to a fixed symmetric and ample line bundle. If $X \subset A$ is an irreducible closed subvariety, $s$ an integer, and $B \in \mathbf{R}$, then

$$
\left\{p \in X^{\mathrm{ta},[s]} \cap A^{[1+s]} ; \hat{h}(p) \leq B\right\}
$$

is finite.

Proof. This result is stated as [8, Corollaire A.1.5, p. 86]. We note that $X(\overline{\mathbf{Q}}) \backslash Z_{X, 0}^{(1+s)}=X^{\mathrm{ta},[s]}$. In fact, Carrizosa's Théorème 20, p. 7 (cf. also Théorème 2.1 [7]) is a relative Lehmer-type height lower bound of sufficient strength in order to apply Rémond's Théorème 2.1 [19] for abelian varieties with complex multiplication.

Corollary 1 follows quickly: forgetting about $\epsilon$ in the bounded height theorem, the height function $\hat{h}$ is bounded on $X^{\mathrm{oa},[s]}(\overline{\mathbf{Q}}) \cap A^{[s]}$. So it is bounded 
on $X^{\mathrm{oa},[s]}(\overline{\mathbf{Q}}) \cap A^{[1+s]}$ too. Finiteness now follows from the proposition since $X^{\mathrm{oa},[s]}(\overline{\mathbf{Q}}) \subset X^{\mathrm{ta},[s]}(\overline{\mathbf{Q}})$.

The proof of Corollary 2 goes as follows: Let $H \subset A$ be an irreducible closed algebraic subgroup, we will show $\operatorname{dim}(X+H)=\min \{\operatorname{dim} X+$ $\operatorname{dim} H, \operatorname{dim} A$ \}. Clearly, the left side is at most the right side. To show the other inequality we fix $\varphi: A \rightarrow A / H$. There is $\psi: A \rightarrow H$ such that $\varphi \times \psi: A \rightarrow(A / H) \times H$ is an isogeny and so $\psi(H)=H$ for dimension reasons. Our hypothesis on $X$ implies $\operatorname{dim} \varphi(X)=\min \{\operatorname{dim} X, \operatorname{dim} A / H\}$. Now the image of $X+H$ under $\varphi \times \psi$ is $\varphi(X) \times H$. Hence $\operatorname{dim}(X+H) \geq$ $\operatorname{dim}(\varphi \times \psi)(X+H)=\min \{\operatorname{dim} X, \operatorname{dim} A / H\}+\operatorname{dim} H$ and our claim follows.

In the proof of Theorem 1.9 [21], Rémond showed that the dimension equality just proved implies $X^{\mathrm{oa}} \neq \emptyset$. Since $X^{\mathrm{oa}}$ is Zariski open the corollary follows from Corollary 1.

Let us consider a power of an elliptic curve with complex multiplication defined over $\overline{\mathbf{Q}}$. Carrizosa's theorem can be proved for such an abelian variety using the semi-relative Lehmer-type height lower bound by Ratazzi [18]. The weaker height bound is compensated by using good bounds by Masser on the cardinality of the torsion points (defined over a number field) of an elliptic curve. Indeed, a related approach was used in [5, Lemma 8.1] by Bombieri, Masser, and Zannier in the toric setting where at the time only a semi-relative Lehmer-type height lower bound was available.

\section{References}

1. Ax, J.: Some topics in differential algebraic geometry I: Analytic subgroups of algebraic groups. Am. J. Math. 94, 1195-1204 (1972)

2. Bombieri, E., Gubler, W.: Heights in Diophantine Geometry. Cambridge University Press, Cambridge (2006)

3. Bombieri, E., Masser, D., Zannier, U.: Intersecting a curve with algebraic subgroups of multiplicative groups. Int. Math. Res. Not. 20, 1119-1140 (1999)

4. Bombieri, E., Masser, D., Zannier, U.: Anomalous subvarieties - structure theorems and applications. Int. Math. Res. Not. 19, 1-33 (2007)

5. Bombieri, E., Masser, D., Zannier, U.: Intersecting a plane with algebraic subgroups of multiplicative groups. Ann. Sc. Norm. Super. Pisa, Cl. Sci. (5) 7, 51-80 (2008)

6. Bombieri, E., Masser, D., Zannier, U.: On unlikely intersections of complex varieties with tori. Acta Arith. (to appear)

7. Carrizosa, M.: Problème de Lehmer et variétés abéliennes CM. C. R. Acad. Sci., Paris, Sér. I (2008). Doi:10.1016/j.crma.2008.10.004

8. Carrizosa, M.: Problème de Lehmer relatif pour les variétés abéliennes CM. Ph.D. thesis, Université Paris 6 (2008)

9. Cassels, J.W.S.: An Introduction to Diophantine Approximation. Cambridge University Press, Cambridge (1965)

10. Danilov, V.I.: Algebraic varieties and schemes. In: Shafarevich, I.R. (ed.) Algebraic Geometry I, Encycl. Math. Sci., vol. 23. Springer, Berlin (1994)

11. Fulton, W.: Intersection Theory. Springer, Berlin (1984)

12. Grauert, H., Remmert, R.: Coherent Analytic Sheaves. Springer, Berlin (1984) 
13. Habegger, P.: On the bounded height conjecture. Int. Math. Res. Not. (to appear)

14. Lang, S.: Fundamentals of Differential Geometry. Springer, New York (2001)

15. Lazarsfeld, R.: Positivity in Algebraic Geometry I. Springer, Berlin (2004)

16. Mumford, D.: Abelian Varieties. Oxford University Press, London (1970)

17. Pink, R.: A common generalization of the conjectures of André-Oort, Manin-Mumford, and Mordell-Lang. Preprint

18. Ratazzi, N.: Intersection de courbes et de sous-groupes et problèmes de minoration de hauteur dans les variétés abéliennes C.M. Ann. Inst. Fourier 58(5), 1575-1633 (2008)

19. Rémond, G.: Intersection de sous-groupes et de sous-variétés I. Math. Ann. 333, 525548 (2005)

20. Rémond, G.: Intersection de sous-groupes et de sous-variétés II. J. Inst. Math. Jussieu 6(2), 317-348 (2007)

21. Rémond, G.: Intersection de sous-groupes et de sous-variétés III. Comment. Math. Helv. (to appear)

22. Schinzel, A.: Polynomials with Special Regard to Reducibility. With an Appendix by Umberto Zannier. Encycl. Math. Appl., vol. 77. Cambridge University Press, Cambridge (2000)

23. Viada, E.: The intersection of a curve with algebraic subgroups in a product of elliptic curves. Ann. Sc. Norm. Super. Pisa, Cl. Sci. (5) 2, 47-75 (2003)

24. Weyl, H.: The Classical Groups. Their Invariants and Representations. Princeton University Press, Princeton, NJ (1973)

25. Whitney, H.: Complex Analytic Varieties. Addison-Wesley, Reading, MA (1972)

26. Zannier, U.: Appendix by Umberto Zannier in [22], pp. 517-539 (2000)

27. Zilber, B.: Exponential sums equations and the Schanuel conjecture. J. Lond. Math. Soc., II. Ser. 65(1), 27-44 (2002) 